\title{
CALIFICACIÓN DEL MÉTODO DEPRONÓSTICO DE TORRES (SEGUNDA PARTE)
}

\author{
Fecha de recepción: 14-07-2009, aprobación: 17-09-2009
}

Miller Jimmy Alarcón

\section{RESUMEN}

Este documento evalúa la precisión de algunos métodos de pronóstico (suavizado por medias móviles, suavizado exponencial simple, suavizado lineal de Holt, suavizado estacional de Winters, suavizado exponencial de Brown y el de suavizado exponencial de Torres) basados en la suavización exponencial y muestra el comportamiento de cada uno de ellos frente a diferentes series de datos históricos.

El método que nos interesa evaluar es el de Torres, para determinar su comportamiento frente otros métodos de suavización exponencial. Se hicieron varias pruebas (usando diferentes series de datos históricos), con el propósito de determinar las bondades o debilidades que este método de pronóstico tiene frente a los demás basados en la suavización exponencial. La prueba es muy sencilla: se toman datos reales y se reservan algunos como datos de prueba. Posteriormente, se comparan con los pronósticos aportados por cada uno de los métodos y de allí se hace una escala para ver cuáles se acercaron más a los datos reales.

\section{ABSTRACT}

This document evaluates the accuracy of some forecasting methods (Smoothing by moving measures, Simple exponential smoothing, Holt's linear smoothing, smoothing and Winters' seasonal exponential smoothing,
Brown's exponential smoothing and Torres' exponential smoothing), based on exponential smoothing shows the behavior of each of the methods evaluated on different sets of historical data.

The method that interests us to evaluate is Torres method, to determine its behavior in other exponential smoothing methods. What was done was to perform several tests (using different sets of historical data), determine the merits or weaknesses of the Torres method against other forecasting methods based on exponential smoothing. The test is simple, real data is taken and some is reserved as proof data, then it is compared with forecasts pooled by each of the methods, from there, a scale is made to see which came closest to the real data.

\section{PALABRAS CLAVE}

Método de Torres, pronóstico, series de tiempo, método Delphi, método Winters, método Brown, tendencia, ciclicidad, estacionalidad, MSE (mínimo error al cuadrado).

\section{KEYWORDS}

Forecast, Time Series, Exponential Smoothing, Delphi Method, Trend, Cyclycity, Seasonal Variation, Winter's Method, Brown's Method, Smoothing Coefficient, Minimum Square Error (MSE). 
Los métodos de media móvil yel de suavización exponencial trabajan con variaciones. En el caso del método de Media móvil, se debe elegir el $n$ (tamaño del subgrupo que se va promediar).

En el caso de suavización exponencial se debe elegir el alpha con el cual se quieren hacerlos cálculos.

\section{INTRODUCCIÓN}

A partir de la investigación desarrollada para evaluar el método de Torres se propuso intensificar más el trabajo de medición y se creyó prudente calificar el desempeño de este método de pronóstico. A continuación se presentan los resultados obtenidos en esta segunda parte de trabajo investigativo.

En este documento se compara el método de Torres (Alarcón, 2005), frente a otros métodos de pronóstico que poseen la misma estructura ${ }^{1}$ es decir, están basados en series de tiempo que emplean la suavización exponencial para su predicción. La prueba consistente en medir la precisión servirá para ver qué tan bueno es este método, de acuerdo con los resultados del pronóstico y midiendo los errores de éste con datos reales reservados para tal fin. De este modo, al confrontarlo con métodos de pronóstico más divulgados se tendrá un ordenamiento que sirva de guía al usuario del método de Torres.

Los resultados de la prueba son meramente informativos. No pretenden imponer uno u otro método, pues para la selección del método de pronóstico hay que tener presente aspectos relacionados con la naturaleza de los datos históricos y las condiciones características de la serie datos, entre otros factores. Por ejemplo, para series que presentan una tendencia clara, se recomienda ${ }^{2}$

\section{RESEÑA DE AUTORES}

Miller Jimmy Alarcón

(Politécnico Grancolombiano)

(malarcon@poligran.edu.co)

El autor de este artículo es Ingeniero Industrial y especialista en Ingeniería de Producción de la Universidad Distrital Francisco José de Caldas. Actualmente se desempeña como director del Departamento Académico de Ingeniería Industrial del Politécnico Grancolombiano y es catedrático de pregrado y posgrado en la Universidad del Rosario y en la Universidad Externado de Colombia. usar métodos como el de Holt o el lineal de Winters. Si la serie presenta estacionalidad clara, se recomienda usar el método estacional de Winters o el método de Brown. Lo que se debe hacer siempre, antes de decidir cuál método de pronóstico usar, es analizar detenidamente la serie para determinar el tipo de comportamiento que tiene, luego hacer una revisión de los diferentes métodos, ver la manera como se ajustan a la serie, revisar los MSE (mínimo error al cuadrado, por sus siglas en inglés) de cada una de los métodos y luego aplicarlo.

\section{MÉTODOS SELECCIONADOS PARA LA PRUEBA}

Existen diferentes métodos de pronóstico conocidos con el nombre de métodos cuantitativos. Como el método de Torres se puede agrupar en esta clasificación, lo enfrentaremos a algunos de los que se encuentran en ella.

Los métodos de media móvil y el de suavización exponencial trabajan con variaciones. En el caso del método de Media móvil, se debe elegir el n (tamaño del subgrupo que se va promediar). En el caso de suavización exponencial se debe elegir el alpha con el cual se quieren hacer los cálculos. Por esta razón se planteó como solución (para ser imparcial y minimizar el error que puedan generar los métodos de pronóstico), trabajar el método de Media móvil con tres diferentes tamaños de subgrupo $=12, \mathrm{n}=63$ el método de suavización exponencial con tres diferentes valores de alpha: alpha 0.3, alpha 0.5 y alpha 0.7.

Para garantizar el mejor desempeño de los métodos de pronóstico se usará en el caso de los métodos de Holt, Brown y Winters, los índices alfa, beta y delta que mejor se ajusten a la serie de datos históricos. Para este propósito se hará uso del 
aplicativo computacional SPSS ${ }^{\circledR 3}$ que automáticamente determina el mejor valor para cada uno de los índices en cada caso.

Para cada una de las pruebas se usarán los siguientes métodos de pronóstico:

I. Media móvil con $\mathrm{N}=12$

II. Media móvil con $\mathrm{N}=6$

III. Media móvil con $\mathrm{N}=3$

IV. Suavización exponencial simple con alpha 0.3

V. Suavización exponencial simple con alpha 0.5

VI. Suavización exponencial simple con alpha 0.7

VII. Método de suavizado lineal de Holt

VIII. Método de suavizado exponencial de Brown

IX. Método de suavizado estacional de Winters

X. Método de Torres ${ }^{4}$ (mejorado con la variación del mayor $\mathrm{R}^{2}$ )

Cabe anotar que los métodos de suavización exponencial simple y Media móvil sirven para pronosticar un solo período, por lo cual es necesario usar los datos pronosticados como si fueran datos históricos, para seguir pronosticando más de un período. En otras palabras, se usarán estos métodos de una forma diferente a la que se conoce en los textos de pronóstico (Makridakis, 1978). Los demás métodos, Torres, Holt, Brown y Winters no tienen esta restricción, porque sirven para pronosticar más de un período.

Descripción de la prueba

El método de prueba usado es bastante simple: se tomó una colección de datos históricos de demanda. A través de los diferentes métodos de pronóstico se trató de ver el comportamiento del método de Torres para lo cual no se usaron todos los datos como históricos sino, que se reservó el último grupo de datos para comparar la dispersión de cada uno de los métodos. Luego se midió el error al restar el dato pronosticado del dato real. Posteriormente se elevó al cuadrado para eliminar la incidencia de resultados negativos y finalmente se sumaron todos los errores elevados al cuadrado. El menor resultado indicó el mejor de los métodos. Adicionalmente, se ordenaron de menor a mayor los valores de los errores al cuadrado para crear el ranking de los métodos. A continuación se pueden ver los pasos de la prueba que corresponden a la técnica del MSE:

I. Recopilar los datos de la serie

II. Reservar datos reales para probar la eficiencia de los métodos

III. Generar el pronóstico con cada uno de los métodos

IV. Medir la dispersión de los pronósticos con respecto de los datos reales

V. Elevar al cuadrado los errores

VI. Sumar los errores al cuadrado de cada método de pronóstico

VII. Ordenar de menor mayor para generar el ranking

\section{BREVE DESCRIPCIÓN DE CADA UNO DE LOS MÉTODOS \\ MEDIA MÓVIIL}

El método de Media móvil consiste en determinar los promedios de los datos históricos a partir del N (tamaño del subgrupo) escogido. Por ejemplo, si se elige un $\mathrm{N}=3$, en una serie de datos reales se pierden los tres primeros datos. Para el cuarto período se toma como pronóstico calculado el promedio de los tres anteriores. Para el quinto período, el dato pronosticado es igual al promedio de los datos históricos de los períodos dos al cuarto y así sucesivamente, hasta terminar con los datos históricos para poder determinar el periodo $t+1$.

Este método:

(...) combina los datos de demanda de la mayor parte de los períodos recien- 
tes, siendo su promedio el pronóstico para el período siguiente. El promedio se "mueve" en el tiempo, en el sentido de que, al transcurrir un período, la demanda del período más antiguo se descarta y se agrega, en su reemplazo, la demanda para el período más reciente, superando así la principal limitación del modelo del promedio simple (Paredes, 2001, p. 10).

El método de Media móvil sirve para pronosticar un solo dato, el del período $t+1$. A pesar de la limitación anterior, usaremos el método de Media móvil introduciendo valores pronosticados para calcular los datos requeridos para la prueba.

A continuación se puede ver el modelo del método de pronóstico:

$\operatorname{MM}($ Media móvil $)=\frac{\sum D_{t}}{n}$

\section{SUAVIZACIÓN EXPONENCIAL SIMPLE}

Paredes menciona que:

(...) la $\operatorname{APICS}^{5}$ ha recomendado el uso de esta técnica para sus asociados. Se distingue porque da pesos de manera exponencial a cada una de las demandas anteriores de calcular el promedio. La demanda de los períodos más recientes recibe un peso mayor; los pesos de los períodos sucesivamente anteriores decaen de una manera no lineal sino exponencial (Paredes, 2001 p.).

El cálculo correspondiente al método de suavización exponencial requiere de dos componentes: el primero es la demanda real del período más reciente. El segundo es el pronóstico más reciente obtenido por este mismo método, es decir el dato pronosticado. Por esta razón, el primer dato se pierde, sólo pronostica del período dos en adelante. Tal como sucede en el caso del método de Media móvil, sirve para pronosticar un solo dato. Pese a esta limitación del método, lo usaremos introduciendo valores pronosticados para calcular los datos requeridos para la prueba.

A continuación se puede ver el modelo del método de pronóstico:

Pronóstico de la demanda del perí́odo $\mathrm{t}+1=\alpha\left(\begin{array}{l}\text { Demanda } \\ \text { más reciente }\end{array}\right)+(1-\alpha) \times\left(\begin{array}{l}\text { Pronóstico } \\ \text { más reciente }\end{array}\right)$
es decir;
\[ F t=\left(\alpha \times D_{t-1}\right)+(1-\alpha) \times S_{t-1} \]
En donde:
$\begin{array}{ll}\mathrm{Ft} \quad & =\text { Pronóstico del período t } \\ \alpha \quad & =\text { Coeficiente de suavización } \\ \mathrm{S}_{\mathrm{t}-1} \quad & =\text { Dato ajustado o calculado del período } t-1 \\ \mathrm{D}_{\mathrm{t}-1} \quad & =\text { Demanda tomada como base para el pronóstico }\end{array}$

\section{MÉTODO DE SUAVIZADO LINEAL DE HOLT}

El método de Holt (Makridakis, 1999), tiene como base la formulación del método de suavización exponencial, pero la ventaja significativa es que permite producir el número de datos pronosticados que se desee, no sólo el siguiente dato $t+1$.

A continuación se puede ver el modelo del método de pronóstico (Pérez, 2005, p. 258) 


$$
\begin{aligned}
& S_{t}=\left(\alpha \times Y_{t}\right)+\left[(1-\alpha) \times\left(S_{t-}\right.\right. \\
& b_{t}=\beta \times\left(S_{t}-S_{t-1}\right)+[(1-\beta) \\
& F_{t+m}=S_{t}+\left(m \times b_{t}\right)
\end{aligned}
$$

En donde:

$\mathrm{S}_{\mathrm{t}}=$ Suavizamiento al finalizar el periodo $\mathrm{t}$

$b_{t}=$ Ajuste de tendencia al finalizar el periodo $t$

$\alpha=$ Constante de alisamiento

$\beta=$ Constante de ajuste de tendencia

$\mathrm{m}=$ Varía desde 1 hasta $\mathrm{m}$, y determina el número de pronósticos que se deseen

$\mathrm{F}_{\mathrm{t}+\mathrm{m}}=$ Pronostico en el periodo $\mathrm{t}+\mathrm{m}$

\section{MÉTODO DE SUAVIZADO EXPONENCIAL DE BROWN}

El método de suavización exponencial de Brown (Pindyck, 2001) produce una serie de datos suavizada a partir de una serie de datos históricos, ya que la nueva serie está constituida por promedios de valores de la serie original. Como en el caso de la suavización exponencial simple es muy importante fijar de manera de correcta el parámetro alpha, entre 0 y 1 . Pérez menciona una regla práctica:
(...) si los datos presentan fuertes fluctuaciones o gran aleatoriedad se deben usar valores de alpha cercanos a 0 ; es decir, que si el parámetro de suavización alpha está próximo a cero, el valor inicial de la serie influirá durante muchos períodos de tiempo. Por el contrario, con valores de alpha próximos a uno, desaparecerá rápidamente la influencia del valor histórico (Pérez, 2005, p. ).

A continuación se presenta la formulación del método de Brown:

$$
\begin{aligned}
& S_{t}^{\prime}=\left(\alpha \times X_{t}\right)+\left[(1-\alpha) \times S_{t-1}^{\prime}\right] \\
& S_{t}^{\prime \prime}=\left(\alpha \times S_{t}^{\prime}\right)+\left[(1-\alpha) \times S_{t-1}^{\prime \prime}\right] \\
& a=S_{t}^{\prime}+\left(S_{t}^{\prime}-S_{t}^{\prime \prime}\right)=2 S_{t}^{\prime}-S_{t}^{\prime \prime} \\
& b_{t}=\frac{\alpha}{1-\alpha}\left(S_{t}^{\prime}-S_{t}^{\prime \prime}\right) \\
& F_{t+m}=a_{t}+b_{t} m
\end{aligned}
$$

\section{Donde:}

$\mathrm{S}_{\mathrm{t}}=$ Valor del Suavizamiento exponencial simple al finalizar el periodo $\mathrm{t}$

$\mathrm{S}_{\mathrm{t}}{ }^{\prime}=$ Valor del suavizamiento exponencial doble al finalizar el período $\mathrm{t}$

$\mathrm{a}_{\mathrm{t}}=$ Ajuste de la serie al finalizar el período $\mathrm{t}$

$\mathrm{b}_{\mathrm{t}}=$ Ajuste de tendencia al finalizar el periodo $\mathrm{t}$

$\alpha=$ Constante de alisamiento

$\beta=$ Constante de ajuste de tendencia

$\mathrm{m}=$ Varía desde 1 hasta $\mathrm{m}$, y determina el número de pronósticos que se deseen $\mathrm{F}_{\mathrm{t}+\mathrm{m}}=$ Pronostico en el periodo $\mathrm{t}+\mathrm{m}$ 


\section{MÉTODO DE SUAVIZADO EXPONENCIAL ESTACIONAL DE WINTERS (PÉREZ, 2005)}

(Pérez, 2005), aclara que el de Winters es un método de pronóstico que generalizó el método de Holt, para tratar con datos que presenten variaciones estacionales. $\mathrm{Al}$ respecto (Makridakis, 1978), menciona que el método de Winters es muy similar al método de Holt, pero a diferencia de éste incluye tres constantes de suavizamiento con valores entre 0 y 1 , una ecuación adicional para trabajar con estacionalidad.

El método de Winters requiere conocer el valor de tres parámetros: alpha, beta y gamma. El primero está relacionado con el componente de aleatoriedad, el segundo con el componente de tendencia de las serie de datos históricos y el tercero con el factor de estacionalidad de la serie. (Makridakis, 1978), aclara que la única manera de conocer el valor de estos tres parámetros es determinado estos valores con ensayo y error, hasta minimizar el MSE. En este documento se harán pruebas en las que, ayudados por programas de computador como SPSS $^{\circledR}$ versión 13.0 y Statgraphics versión 5.1, se determinará automáticamente el valor de estos tres parámetros.

A continuación se puede ver la formulación del método de Winters:

$$
\begin{aligned}
& S_{t}=\alpha \frac{X_{t}}{I_{t-L}}+(1-\alpha)\left(S_{t-1}+b_{t-1}\right) \\
& b_{t}=\gamma\left(S_{t}-S_{t-1}\right)+(1-\gamma) b_{t-1} \\
& I_{t}=\beta \frac{X_{t}}{S_{t}}+(1-\beta) I_{t-L} \\
& F_{t+m}=\left(S_{t}+b_{t} m\right) I_{t-L+m}
\end{aligned}
$$

En donde:

$\mathrm{S}_{\mathrm{t}}=$ Valor del Suavizamiento al finalizar el periodo $\mathrm{t}$

$\mathrm{b}_{\mathrm{t}}=$ Ajuste de tendencia al finalizar el periodo $\mathrm{t}$

$\alpha=$ Constante de alisamiento

$\beta=$ Constante de ajuste de tendencia

$\gamma=$ Constante de ajuste a la estacionalidad

$\mathrm{L}=$ Longitud de la estacionalidad (por ejemplo número de meses o trimestres)

$\mathrm{I}=$ Factor de ajuste de la estacionalidad

$\mathrm{m}=$ Varía desde 1 hasta $\mathrm{m}$, $\mathrm{y}$ determina el número de pronósticos que se deseen $\mathrm{F}_{\mathrm{t}+\mathrm{m}}=$ Pronostico en el periodo $\mathrm{t}+\mathrm{m}$

\section{MÉTODO DE PRONÓSTICO DE TORRES}

El método de Torres presenta la siguiente metodología ${ }^{6}$ :

a. Cálculo del pronóstico simple;

$$
\mathrm{S}_{\mathrm{t}+1}=\alpha \times X_{t}+(1-\alpha) \times \mathrm{S}_{\mathrm{t}}
$$

Donde $\mathrm{X}_{\mathrm{t}}$ se refiere a la demanda histórica en cada período de tiempo: 
b. Cálculo del pronóstico doble;

$$
\mathrm{S}_{\mathrm{t}+1}=\alpha \times S_{t+1}+(1-\alpha) \times \mathrm{S}_{\mathrm{t}}
$$

c. Cálculo del pronóstico de ajuste;

$$
\begin{aligned}
& S_{t+1}^{\wedge}=a+b \\
& a=\left(2 \times S_{t+1}\right)-S_{t+1}^{\prime} \\
& \mathrm{b}=\frac{\alpha}{1-\alpha} \times\left(\mathrm{S}_{\mathrm{t}+1}-\mathrm{S}_{\mathrm{t}+1}^{\prime}\right) \\
& \mathrm{b}=\frac{\alpha}{1-\alpha} \times\left(\mathrm{S}_{\mathrm{t}+1}-\mathrm{S}_{\mathrm{t}+1}^{\prime}\right)
\end{aligned}
$$

Introduce un comportamiento heurístico que determina el porcentaje de crecimiento o decrecimiento

\section{d. Pronósticos alternos}

El método de Torres trabaja tres planes alternos: demanda alta, demanda baja y demanda constante. Para hallar los valores de los planes alternos: demanda alta, baja y constante se debe buscar, para cada uno de los períodos dentro de los datos ajustados (pronóstico simple y doble pronóstico de ajuste), el valor más alto, que lo toma como el pronóstico alterno de demanda alta, el valor más bajo, que se refiere al pronóstico alterno de demanda baja y el valor más cercano al dato histórico, que recibe el nombre de pronóstico alterno de demanda constante.

\section{f. Demanda Agregada Global:}

$$
\mathrm{DAG}_{\mathrm{t}}=\mathrm{D}_{\mathrm{t}} \times(1+\% \mathrm{P})
$$

Donde;

$\mathrm{D}_{\mathrm{t}}=$ dato más cercano al histórico en cada período

$\% \mathrm{P}=$ Crecimiento o decrecimiento ${ }^{7}$ general de la demanda para el siguiente período, si es decrecimiento debe usarse en la formula $-\% \mathrm{P}$.

\section{g. Índice de Aseguramiento:}

$\mathrm{IA}_{\mathrm{t}+1}=\mathrm{X}_{\mathrm{t}+1} / \mathrm{X}_{\mathrm{t}}$

Donde:

$\mathrm{IA}_{\mathrm{t}}=$ Índice de Aseguramiento del comportamiento de la variable en el período $\mathrm{t}$

$\mathrm{X}_{\mathrm{t}}=$ Demanda histórica o variable de entrada en el período $\mathrm{t}$ general de la demanda para el período siguiente. 


\section{h. Pronóstico Final DAT ${ }^{8}$ :}

$\mathrm{DAT}_{\mathrm{i}=1, \mathrm{n}}=\mathrm{DAG}_{\mathrm{t}} \times \mathrm{IA}_{\mathrm{t}}$

Donde;

DAT $_{i}=$ Demanda Agregada Total del período $i$, se refiere al valor pronosticado través del método, para los períodos siguientes.

$\mathrm{DAG}_{\mathrm{t}}=$ Demanda Agregada Global del período $\mathrm{t}$

$\mathrm{IA}_{\mathrm{t}}=$ Índice de aseguramiento

\section{PRUEBA NÚMERO UNO}

La primera serie de datos está compuesta por ciento ocho datos de demandas mensuales (Vélez, 2004) es decir, nueve años. Los datos tomados como históricos son noventa y seis y los últimos doce datos se reservan para probar la eficiencia de los métodos usados.

Los noventa y seis datos históricos son los siguientes:

\begin{tabular}{||l|r|r|r|r|r|r|r|r||}
\hline \multicolumn{1}{|c|}{ Mes } & Año 1 & Año 2 & Año 3 & Año 4 & Año 5 & Año 6 & Año 7 & \multicolumn{1}{c|}{ Año 8 } \\
\hline Enero & 1738 & 2224 & 2939 & 4009 & 5236 & 7242 & 10058 & 13959 \\
\hline Febrero & 1983 & 2459 & 3320 & 4577 & 6037 & 7955 & 10997 & 14873 \\
\hline Marzo & 2240 & 2657 & 3623 & 5039 & 6608 & 9149 & 12133 & 16568 \\
\hline Abril & 2138 & 2858 & 3765 & 4717 & 6426 & 8670 & 12182 & 16972 \\
\hline Mayo & 2330 & 2832 & 3812 & 4969 & 6614 & 9561 & 12916 & 17023 \\
\hline Junio & 2333 & 2782 & 4157 & 5283 & 6986 & 9636 & 13032 & 17237 \\
\hline Julio & 2320 & 3025 & 4100 & 5209 & 7017 & 9790 & 13379 & 18034 \\
\hline Agosto & 2364 & 3002 & 4135 & 5250 & 7322 & 10182 & 13955 & 17789 \\
\hline Septiembre & 2463 & 3151 & 4220 & 5597 & 7658 & 10441 & 14185 & 18168 \\
\hline Octubre & 2579 & 3283 & 4313 & 5449 & 7661 & 11184 & 14984 & 19031 \\
\hline Noviembre & 2545 & 3355 & 4440 & 5704 & 8070 & 11562 & 14825 & 18234 \\
\hline Diciembre & 4091 & 5296 & 6909 & 9154 & 10877 & 14628 & 18964 & 24379 \\
\hline \hline
\end{tabular}

Los doce datos reservados para hacer la comparación son los siguientes:

\begin{tabular}{||l|l||}
\hline \multicolumn{1}{|c|}{ Mes } & Año 9 \\
\hline Enero & 17176 \\
\hline Febrero & 18327 \\
\hline Marzo & 21819 \\
\hline Abril & 19627 \\
\hline Mayo & 20969 \\
\hline Junio & 22046 \\
\hline Julio & 22068 \\
\hline Agosto & 21949 \\
\hline Septiembre & 22630 \\
\hline Octubre & 23609 \\
\hline Noviembre & 22573 \\
\hline Diciembre & 29583 \\
\hline
\end{tabular}

En la siguiente gráfica se puede ver el comportamiento de la serie de datos de las demandas mensuales. Así mismo, se puede ver claramente la tendencia de la variable y su estacionalidad. La ciclicidad, por ser un elemento de largo plazo, no puede constatarse debido al poco número de datos. 


\section{Demandas}

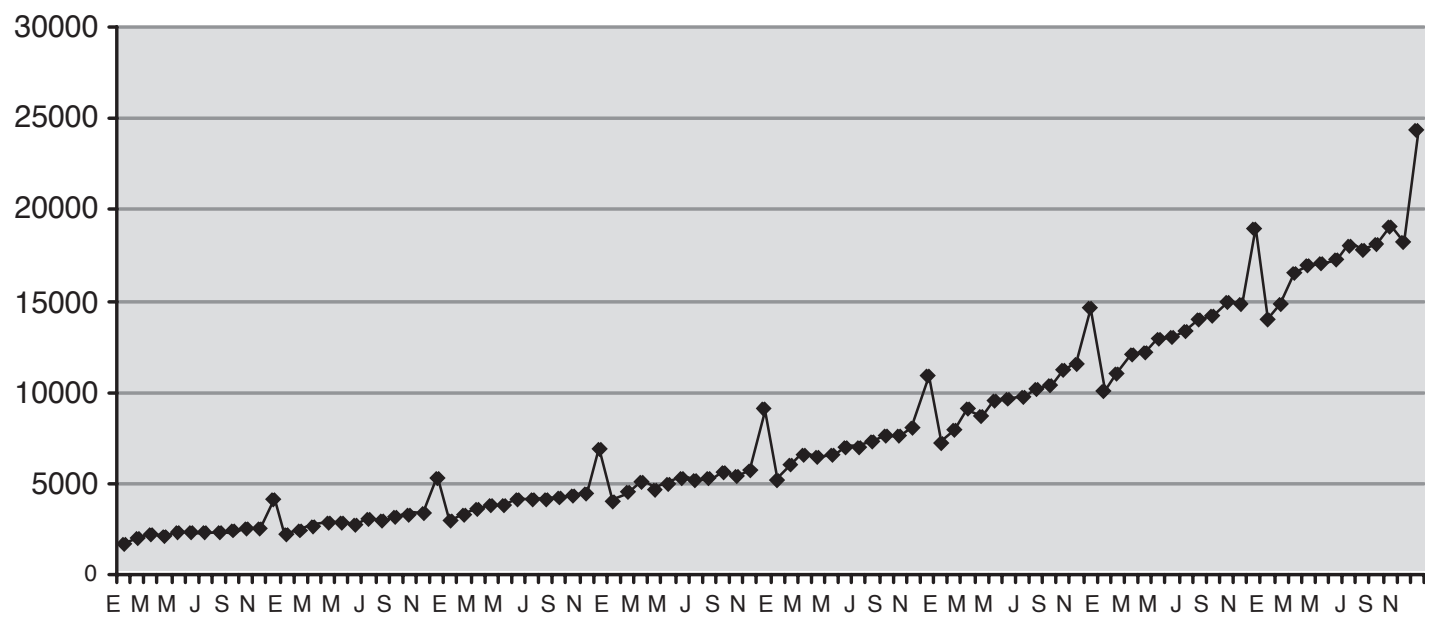

Gráfica 1. Comportamiento de los 96 datos históricos para la prueba número uno

Cabe recordar que para generar los pronósticos se usaron dos paquetes computacionales diseñados para este fin. Para los pronósticos de Winters se usó SPSS ${ }^{\circledR}$ versión 13.0. Para los pronósticos de Holt y Brown se usó el paquete Statgraphics versión 5.1. Estos paquetes eliminan el trabajo de hallar los mejores índices $\alpha, \gamma$ y $\beta$ que requiere cada uno de los métodos para generar los pronósticos.

A continuación pueden verse los resultados de la ejecución del programa Statgraphics para la prueba número uno. En ellos se observa el valor de los índices $\alpha, \gamma$ y $\beta$ determinados automáticamente con la ayuda de este software:

\section{Gráfico de Secuencia de tiempo para Demandas}

(X 10000)

Suavizado exp. lineal de Holt con alfa $=0,0874$ y beta $=0,253$

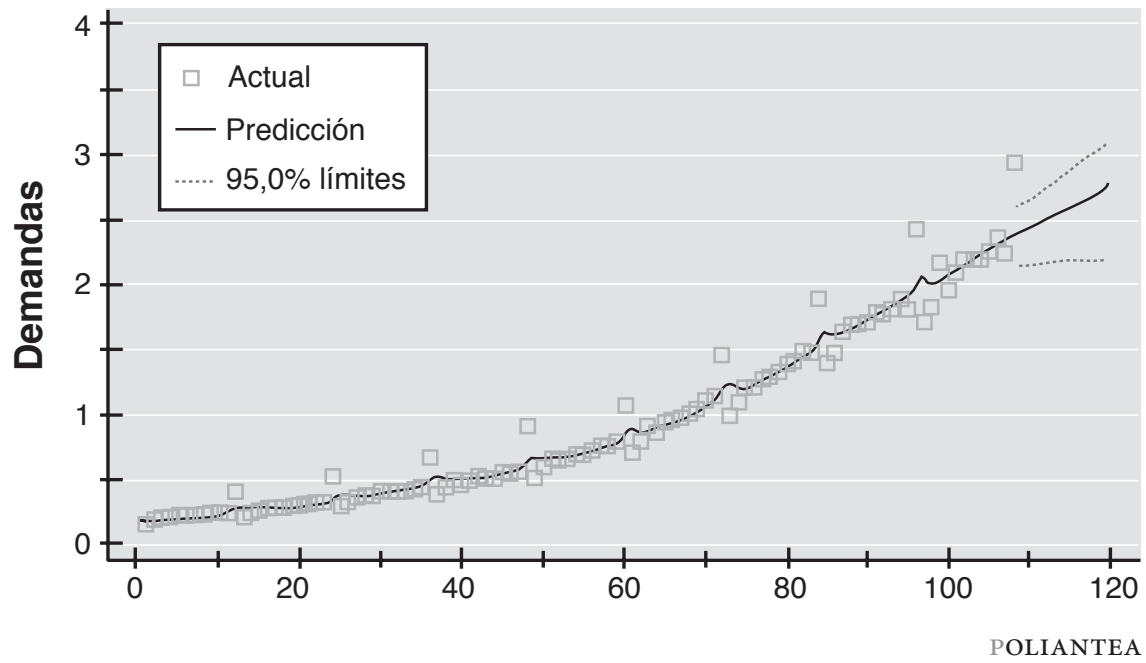




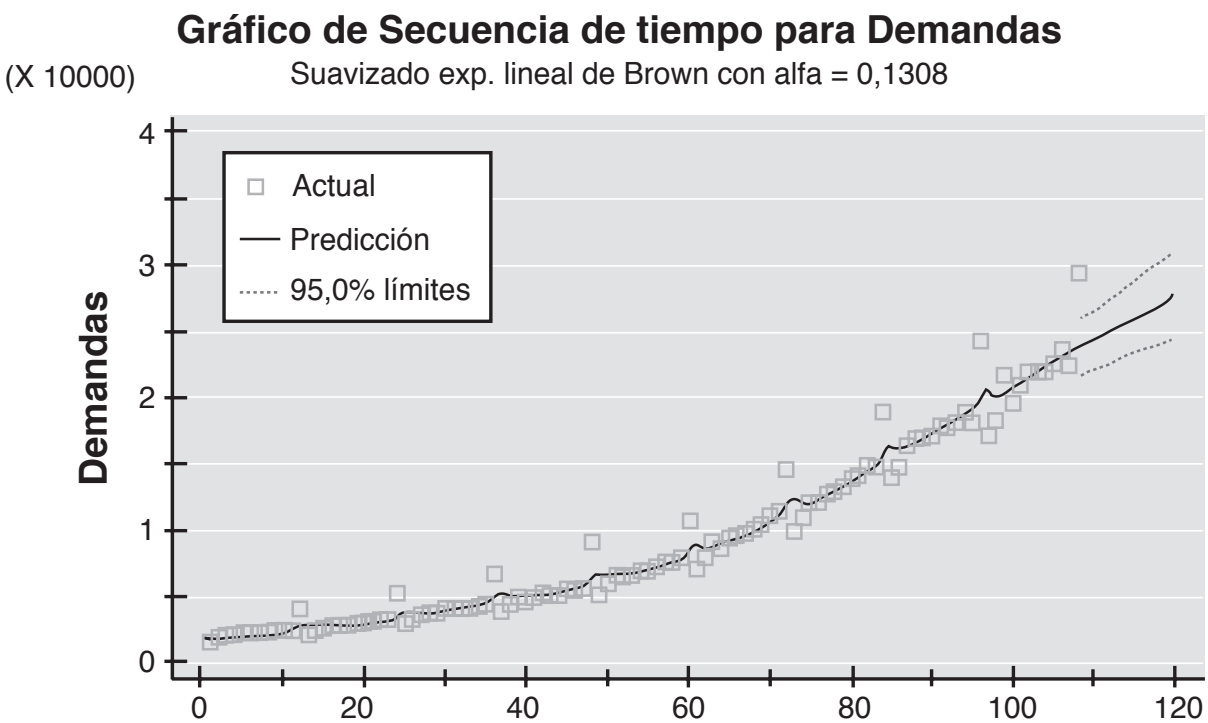

Los resultados de los pronósticos usando cada uno de los métodos son los siguientes:

\begin{tabular}{|c|c|c|c|c|c|c|c|}
\hline \multirow{2}{*}{ Per } & \multirow{2}{*}{$\begin{array}{c}\text { Demanda } \\
\text { Real }\end{array}$} & \multicolumn{3}{|c|}{ Media Móvil } & \multirow{2}{*}{$\begin{array}{l}\text { Método } \\
\text { de } \\
\text { Brown }\end{array}$} & \multirow{2}{*}{$\begin{array}{l}\text { Método } \\
\text { de } \\
\text { Winters }\end{array}$} & \multirow{2}{*}{$\begin{array}{l}\text { Método } \\
\text { Holt }\end{array}$} \\
\hline & & $n=12$ & $\mathrm{n}=6$ & $\mathrm{n}=3$ & & & \\
\hline Ene & 17176 & 17689 & 16105 & 15133 & 19417 & 17510 & 24560 \\
\hline Feb & 18327 & 18028 & 16785 & 16138 & 19725 & 18502 & 25022 \\
\hline Mar & 21819 & 18344 & 17271 & 16854 & 20033 & 20430 & 25485 \\
\hline Abr & 19627 & 18541 & 17537 & 17077 & 20341 & 20530 & 25947 \\
\hline May & 20969 & 18737 & 17880 & 17431 & 20649 & 20535 & 26410 \\
\hline Jun & 22046 & 18982 & 18082 & 17687 & 20957 & 20657 & 26872 \\
\hline Jul & 22068 & 19273 & 19273 & 17997 & 21265 & 21288 & 27335 \\
\hline Ago & 21949 & 19520 & 19520 & 18329 & 21573 & 21013 & 27797 \\
\hline Sep & 22630 & 19953 & 19953 & 18478 & 21881 & 21411 & 28260 \\
\hline Oct & 23609 & 20548 & 20548 & 20548 & 22189 & 22396 & 28723 \\
\hline Nov & 22573 & 21307 & 21307 & 21307 & 22497 & 21654 & 29185 \\
\hline Dic & 29583 & 24379 & 24379 & 24379 & 22805 & 28822 & 29648 \\
\hline
\end{tabular}




\begin{tabular}{||c|c|c|c|c|c||}
\hline \multirow{2}{*}{ Per } & \multirow{2}{*}{$\begin{array}{c}\text { Demanda } \\
\text { Real }\end{array}$} & \multicolumn{2}{|c|}{ Suavización exponencial simple } & \\
\cline { 5 - 6 } & & Alpha = 0,5 & Alpha = 0,7 & Alpha = 0,3 & $\begin{array}{c}\text { Método } \\
\text { Torres }\end{array}$ \\
\hline Ene & 17176 & 21604 & 22819 & 20186 & 18547 \\
\hline Feb & 18327 & 21842 & 23057 & 20424 & 19888 \\
\hline Mar & 21819 & 22080 & 23295 & 20663 & 20758 \\
\hline Abr & 19627 & 22319 & 23534 & 20901 & 21832 \\
\hline May & 20969 & 22557 & 23772 & 21139 & 21663 \\
\hline Jun & 22046 & 22795 & 24010 & 21378 & 22112 \\
\hline Jul & 22068 & 23034 & 24249 & 21616 & 23145 \\
\hline Ago & 21949 & 23272 & 24487 & 21854 & 21908 \\
\hline Sep & 22630 & 23510 & 24725 & 22093 & 23307 \\
\hline Oct & 23609 & 23749 & 24964 & 22331 & 24305 \\
\hline Nov & 22573 & 23987 & 25202 & 22569 & 21608 \\
\hline Dic & 29583 & 24225 & 25440 & 22808 & 31733 \\
\hline \hline
\end{tabular}

Errores en unidades: en la siguiente que tuvieron los pronósticos de cada uno de tabla se pueden ver los errores en unidades los métodos frente al dato histórico.

\begin{tabular}{|c|c|c|c|c|c|c|c|c|c|c|c|}
\hline \multirow{2}{*}{ Per } & \multirow{2}{*}{$\begin{array}{l}\text { Demanda } \\
\text { Real }\end{array}$} & \multicolumn{3}{|c|}{ Media Móvil } & \multirow{2}{*}{$\begin{array}{l}\text { Método } \\
\text { de } \\
\text { Brown }\end{array}$} & \multirow{2}{*}{$\begin{array}{l}\text { Método } \\
\text { de } \\
\text { Winters }\end{array}$} & \multirow{2}{*}{$\begin{array}{c}\text { Método } \\
\text { de } \\
\text { Holt }\end{array}$} & \multicolumn{3}{|c|}{ Suavización exponencial simple } & \multirow{2}{*}{$\begin{array}{l}\text { Método } \\
\text { Torres }\end{array}$} \\
\hline & & $n=12$ & $n=6$ & $\mathrm{n}=3$ & & & & Alpha $=0,5$ & Alpha $=0,7$ & Alpha $=0,3$ & \\
\hline Ene & 17.176 & 513 & 1.071 & 2.043 & 2.241 & 334 & 7.384 & 4.428 & 5.643 & 3.010 & 1.371 \\
\hline Feb & 18.327 & 299 & 1.543 & 2.189 & 1.398 & 175 & 6.695 & 3.515 & 4.730 & 2.097 & 1.561 \\
\hline Mar & 21.819 & 3.476 & 4.549 & 4.965 & 1.786 & 1.389 & 3.666 & 261 & 1.476 & 1.156 & 1.061 \\
\hline Abr & 19.627 & 1.086 & 2.090 & 2.550 & 714 & 903 & 6.320 & 2.692 & 3.907 & 1.274 & 2.205 \\
\hline May & 20.969 & 2.232 & 3.089 & 3.538 & 320 & 434 & 5.441 & 1.588 & 2.803 & 170 & 694 \\
\hline Jun & 22.046 & 3.064 & 3.964 & 4.359 & 1.089 & 1.389 & 4.826 & 749 & 1.964 & 668 & 66 \\
\hline Jul & 22.068 & 2.796 & 2.796 & 4.071 & 803 & 780 & 5.267 & 966 & 2.181 & 452 & 1.077 \\
\hline Ago & 21.949 & 2.429 & 2.429 & 3.620 & 376 & 936 & 5.848 & 1.323 & 2.538 & 95 & 41 \\
\hline Sep & 22.630 & 2.677 & 2.677 & 4.152 & 749 & 1.219 & 5.630 & 880 & 2.095 & 537 & 677 \\
\hline Oct & 23.609 & 3.061 & 3.061 & 3.061 & 1.420 & 1.213 & 5.114 & 140 & 1.355 & 1.278 & 696 \\
\hline Nov & 22.573 & 1.267 & 1.267 & 1.267 & 76 & 919 & 6.612 & 1.414 & 2.629 & 4 & 965 \\
\hline Dic & 29.583 & 5.204 & 5.204 & 5.204 & 6.778 & 761 & 65 & 5.358 & 4.143 & 6.775 & 2.150 \\
\hline
\end{tabular}

Con este método de prueba se mide el menor error cuadrático partiendo de la diferencia entre el dato pronosticado menos la demanda y ese resultado elevado al cuadrado. Con la suma de todos los errores al cuadrado $\mathrm{RSE}^{9}$ se puede determinar cuál estuvo más cerca del dato real. Los valores de las diferencias al cuadrado se muestran continuación: 


\begin{tabular}{|c|c|c|c|c|c|c|c|}
\hline \multirow{2}{*}{ Per } & \multirow{2}{*}{$\begin{array}{c}\text { Demanda } \\
\text { Real }\end{array}$} & \multicolumn{3}{|c|}{ Media Móvil } & \multirow{2}{*}{$\begin{array}{l}\text { Método de } \\
\text { Brown }\end{array}$} & \multirow{2}{*}{$\begin{array}{l}\text { Método de } \\
\text { Winters }\end{array}$} & \multirow{2}{*}{$\begin{array}{c}\text { Método de } \\
\text { Holt }\end{array}$} \\
\hline & & $n=12$ & $n=6$ & $\mathbf{n}=\mathbf{3}$ & & & \\
\hline Ene & 17.176 & 263.084 & 1.146 .327 & 4.172 .487 & 5.022 .081 & 111.556 & 54.523 .456 \\
\hline Feb & 18.327 & 89.401 & 2.379 .306 & 4.793 .180 & 1.954 .404 & 30.625 & 44.823 .025 \\
\hline Mar & 21.819 & 12.079 .100 & $2^{0.688 .852}$ & 24.647 .915 & 3.189 .796 & 1.929 .321 & 13.439 .556 \\
\hline Abr & 19.627 & 1.179 .879 & 4.367 .403 & 6.500 .800 & 509.796 & 815.409 & 39.942 .400 \\
\hline May & 20.969 & 4.982 .382 & 9.539 .862 & 12.515 .085 & 102.400 & 188.356 & 29.604 .481 \\
\hline Jun & 22.046 & 9.389 .847 & 15.711 .975 & 19.003 .787 & 1.185 .921 & 1.929 .321 & 23.290 .276 \\
\hline Jul & 22.068 & 7.814 .820 & 7.814 .820 & 16.573 .041 & 644.809 & 608.400 & 27.741 .289 \\
\hline Ago & 21.949 & 5.899 .069 & 5.899 .069 & 13.101 .987 & 141.376 & 876.096 & 34.199 .104 \\
\hline Sep & 22.630 & 7.166 .329 & 7.166 .329 & 17.241 .872 & 561.001 & 1.485 .961 & 31.696 .900 \\
\hline Oct & 23.609 & 9.369 .721 & 9.369 .721 & 9.369 .721 & 2.016 .400 & 1.471 .369 & 26.152 .996 \\
\hline Nov & 22.573 & 1.604 .022 & 1.604 .022 & 1.604 .022 & 5.776 & 844.561 & 43.718 .544 \\
\hline Dic & 29.583 & 27.081 .616 & 27.081 .616 & 27.081 .616 & 45.941 .284 & 579.121 & 4.225 \\
\hline \multicolumn{2}{|c|}{ Total } & 86.919 .270 & 112.769 .303 & 156.605 .514 & 61.275 .044 & 10.870 .096 & 369.136 .252 \\
\hline
\end{tabular}

\begin{tabular}{|c|c|c|c|c|c|}
\hline \multirow[b]{2}{*}{ Per } & \multirow[b]{2}{*}{$\begin{array}{c}\text { Demanda } \\
\text { Real }\end{array}$} & \multicolumn{3}{|c|}{ Suavización exponencial simple } & \multirow[b]{2}{*}{ Método Torres } \\
\hline & & $\begin{array}{c}\text { Alpha }= \\
0,5\end{array}$ & Alpha $=0,7$ & Alpha $=0,3$ & \\
\hline Ene & 17.176 & 19.604 .056 & 31.843 .449 & 9.060 .100 & 1.879 .641 \\
\hline Feb & 18.327 & 12.355 .036 & 22.372 .900 & 4.397.409 & 2.436 .721 \\
\hline Mar & 21.819 & 68.277 & 2.178 .576 & 1.336 .336 & 1.125 .721 \\
\hline Abr & 19.627 & 7.244 .849 & 15.264 .649 & 1.623 .076 & 4.862 .025 \\
\hline May & 20.969 & 2.521 .592 & 7.856 .809 & 28.900 & 481.636 \\
\hline Jun & 22.046 & 561.418 & 3.857 .296 & 446.224 & 4.356 \\
\hline Jul & 22.068 & 932.392 & 4.756 .761 & 204.304 & 1.159 .929 \\
\hline Ago & 21.949 & 1.750 .147 & 6.441 .444 & 9.025 & 1.681 \\
\hline Sep & 22.630 & 774.853 & 4.389.025 & 288.369 & 458.329 \\
\hline Oct & 23.609 & 19.484 & 1.836 .025 & 1.633 .284 & 484.416 \\
\hline Nov & 22.573 & 1.999 .141 & 6.911 .641 & 16 & 931.225 \\
\hline Dic & 29.583 & 28.705 .632 & 17.164 .449 & 45.900 .625 & 4.622 .500 \\
\hline \multicolumn{2}{|c|}{ Total } & 76.536 .878 & 124.873 .024 & 64.927 .668 & 18.448 .180 \\
\hline
\end{tabular}


Los resultados de la clasificación son los siguientes:

\begin{tabular}{|l|l|r|r||}
\hline Ranking & \multicolumn{1}{|c|}{ Método } & \multicolumn{1}{c|}{$\begin{array}{c}\text { Suma Errores } \\
\text { cuadrado }\end{array}$} & $\begin{array}{l}\text { Desviación } \\
\text { Porcentual }\end{array}$ \\
\hline Primero & Winters & 10.870 .096 & $0,00 \%$ \\
\hline Segundo & Torres & $\mathbf{1 8 . 4 4 8 . 1 8 0}$ & $\mathbf{6 9 , 7 1 \%}$ \\
\hline Tercero & Brown & 61.275 .044 & $463,70 \%$ \\
\hline Cuarto & Suavización Alpha 0,3 & 64.927 .668 & $497,31 \%$ \\
\hline Quinto & Suavización Alpha 0,5 & 76.536 .878 & $604,10 \%$ \\
\hline Sexto & Promedio Móvil N = 12 & 86.919 .270 & $699,62 \%$ \\
\hline SĖptimo & Promedio Móvil N =6 & 112.769 .303 & $937,43 \%$ \\
\hline Octavo & Suavización Alpha 0,7 & 124.873 .024 & $1048,78 \%$ \\
\hline Noveno & Promedio Móvil N = 3 & 156.605 .514 & $1340,70 \%$ \\
\hline Décimo & Holt & 369.136 .252 & $3295,89 \%$ \\
\hline
\end{tabular}

PRUEBA MÚMERO OOS:

Para realizar una segunda prueba se eligió una nueva serie de datos que representa el consumo promedio de kilovatios de energía eléctrica en Colombia ${ }^{10}$ en los últi- mos seis años es decir, una serie de setenta y dos datos. Los últimos doce datos se reservan para probar la eficiencia de los métodos usados. A continuación pueden verse los datos originales:

\begin{tabular}{||l|c|c|c|c|c|c||}
\hline \multicolumn{7}{||c||}{ Demanda energía promedio } \\
\hline Período & $\mathbf{1 9 9 9}$ & $\mathbf{2 0 0 0}$ & $\mathbf{2 0 0 1}$ & $\mathbf{2 0 0 2}$ & $\mathbf{2 0 0 3}$ & $\mathbf{2 0 0 4}$ \\
\hline ENERO & 117,8 & 114,6 & 119,1 & 122,3 & 127,8 & 128,6 \\
\hline FEBRERO & 120,1 & 119,7 & 122,3 & 127,2 & 130,9 & 134,0 \\
\hline MARZO & 119,0 & 119,1 & 122,5 & 126,3 & 131,4 & 135,3 \\
\hline ABRIL & 119,1 & 119,4 & 122,4 & 124,8 & 129,5 & 133,0 \\
\hline MAYO & 119,2 & 119,9 & 122,7 & 127,2 & 130,1 & 133,2 \\
\hline JUNIO & 118,0 & 120,7 & 122,0 & 125,4 & 128,0 & 133,6 \\
\hline JULIO & 118,4 & 121,7 & 122,6 & 125,4 & 129,8 & 131,8 \\
\hline AGOSTO & 118,9 & 121,8 & 126,3 & 127,9 & 131,5 & 135,1 \\
\hline SEPTIEMBRE & 118,4 & 121,3 & 125,6 & 129,1 & 131,7 & 133,5 \\
\hline OCTUBRE & 119,9 & 121,5 & 126,2 & 129,0 & 131,8 & 133,8 \\
\hline NOVIEMBRE & 121,0 & 123,6 & 124,7 & 130,1 & 133,5 & 135,1 \\
\hline DICIEMBRE & 118,8 & 124,9 & 125,3 & 130,1 & 132,4 & 134,9 \\
\hline
\end{tabular}

Los doce datos reservados para hacer la comparación son los siguientes:

\begin{tabular}{|l|c|l|c|}
\hline \multicolumn{1}{|c|}{ Mes } & Año 9 & \multicolumn{1}{c|}{ Mes } & Año 9 \\
\hline Enero & 128,6 & Julio & 131,8 \\
\hline Febrero & 134,0 & Agosto & 135,1 \\
\hline Marzo & 135,3 & Septiembre & 133,5 \\
\hline Abril & 133,0 & Octubre & 133,8 \\
\hline Mayo & 133,2 & Noviembre & 135,1 \\
\hline Junio & 133,6 & Diciembre & 134,9 \\
\hline
\end{tabular}

En la siguiente gráfica se puede ver el comportamiento de la serie de datos de los consumos mensuales de energía eléctrica de nuestro país: 


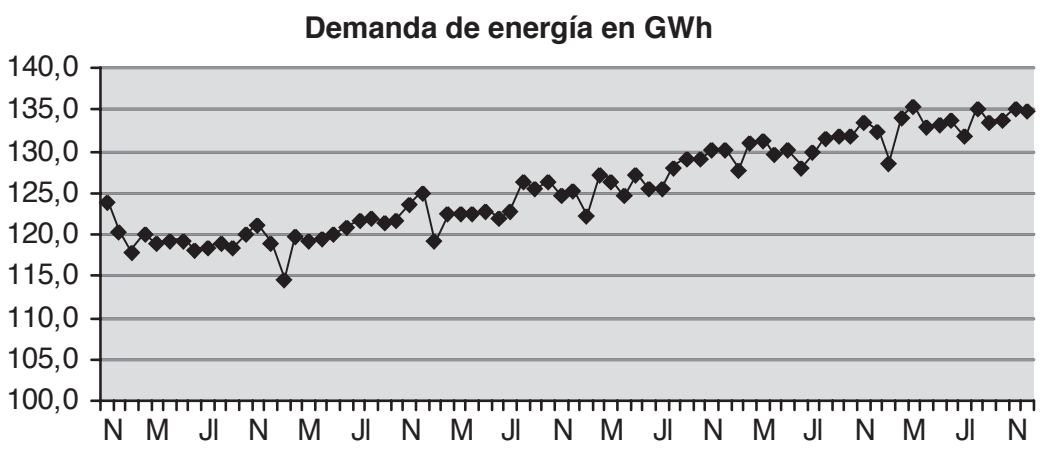

Gráfica 2. Comportamiento de los 72 datos históricos para la prueba número dos

A continuación se muestran valores de los índices $\alpha, \gamma$ y $\beta$ deterlos resultados de la ejecución del minados automáticamente con la programa Statgraphics ${ }^{\circledR}$ junto a los ayuda de este software:

\section{Gráfico de Secuencia de tiempo para Energía}

Suavizado exp. lineal de Brown con alfa $=0,1391$

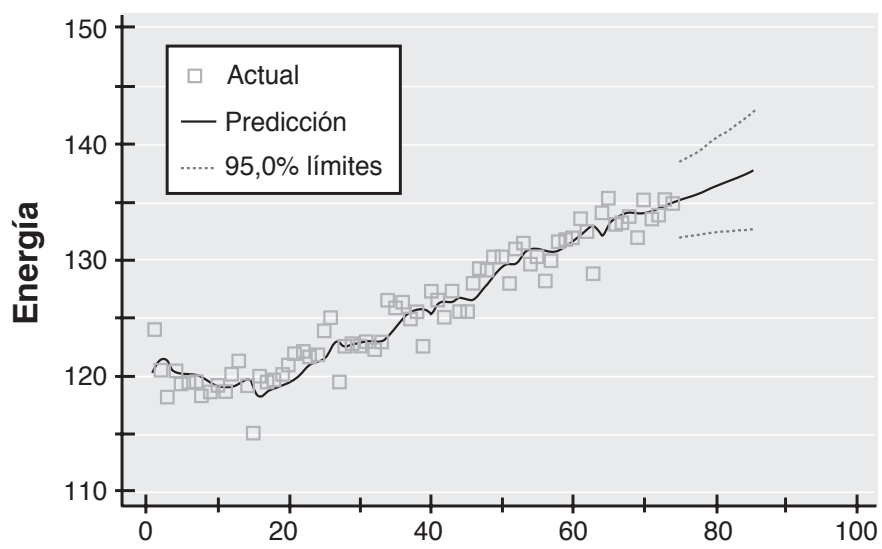

\section{Gráfico de Secuencia de tiempo para Energía}

Suavizado exp. lineal de Holt con alfa $=0,234$ y beta $=0,1115$

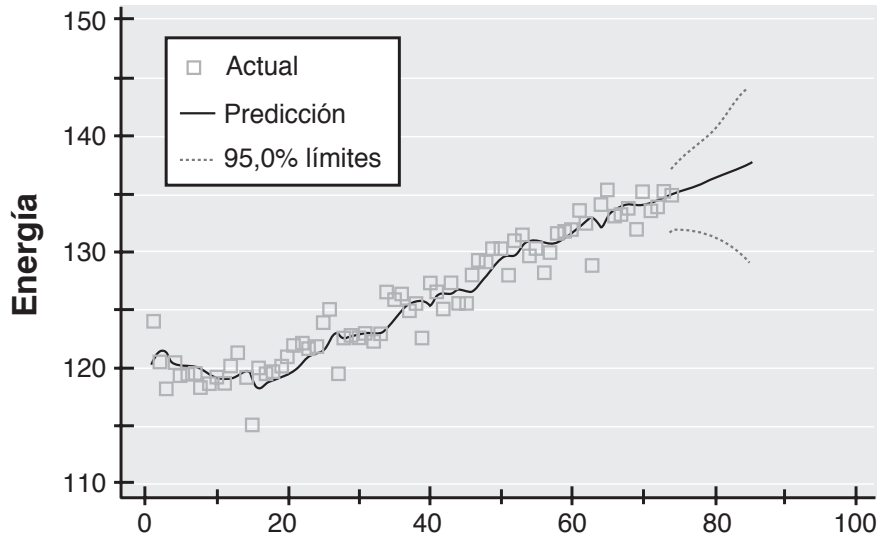


Los resultados de los pronósticos usando cada uno de los métodos son los siguientes:

\begin{tabular}{|c|c|c|c|c|c|c|c|c|c|c|c|}
\hline \multirow{2}{*}{ Per } & \multirow{2}{*}{$\begin{array}{l}\text { Demanda } \\
\text { real }\end{array}$} & \multicolumn{3}{|c|}{ Media Móvil } & \multirow{2}{*}{$\begin{array}{c}\text { Método } \\
\text { de } \\
\text { Brown }\end{array}$} & \multirow{2}{*}{$\begin{array}{l}\text { Método } \\
\text { de } \\
\text { Winters }\end{array}$} & \multirow{2}{*}{$\begin{array}{l}\text { Método } \\
\text { de Holt }\end{array}$} & \multicolumn{3}{|c|}{ Suavización exponencial simple } & \multirow{2}{*}{$\begin{array}{l}\text { Método } \\
\text { Torres }\end{array}$} \\
\hline & & $n=12$ & $n=6$ & $n=3$ & & & & Alpha $=0,3$ & Alpha $=0,5$ & Alpha $=0,7$ & \\
\hline Ene & 128,6 & 130,5 & 129,9 & 129,3 & 132,9 & 128,7 & 132,851 & 132 & 132,6 & 132,7 & 131 \\
\hline Feb & 134 & 130,7 & 130 & 129,6 & 133,2 & 133,1 & 133,169 & 132,2 & 132,7 & 132,9 & 138,6 \\
\hline Mar & 135,3 & 131 & 129,6 & 130 & 133,5 & 132,6 & 133,487 & 132,3 & 132,9 & 133 & 134,22 \\
\hline Abr & 133 & 131 & 129,9 & 130,6 & 133,9 & 132,0 & 133,805 & 132,5 & 133 & 133,1 & 130,79 \\
\hline May & 133,2 & 130,9 & 130 & 130,3 & 134,2 & 132,7 & 134,122 & 132,6 & 133,1 & 133,3 & 133,19 \\
\hline Jun & 133,6 & 131,1 & 130,1 & 129,2 & 134,5 & 131,7 & 134,44 & 132,8 & 133,3 & 133,4 & 134,44 \\
\hline Jul & 131,8 & 131,2 & 130,5 & 129,3 & 134,8 & 132,4 & 134,758 & 132,9 & 133,4 & 133,6 & 131,13 \\
\hline Ago & 135,1 & 131,8 & 131 & 129,7 & 135,1 & 134,1 & 135,075 & 133 & 133,6 & 133,7 & 137,43 \\
\hline Sep & 133,5 & 132,2 & 131,8 & 131 & 135,4 & 134,4 & 135,393 & 133,2 & 133,7 & 133,8 & 132,4 \\
\hline Oct & 133,8 & 132,4 & 132,2 & 131,7 & 135,7 & 135,0 & 135,711 & 133,3 & 133,9 & 134 & 134,28 \\
\hline Nov & 135,1 & 132,6 & 132,4 & 132,3 & 136,1 & 135,9 & 136,029 & 133,5 & 134 & 134,1 & 136,35 \\
\hline Dic & 134,9 & 133 & 132,6 & 132,6 & 136,4 & 135,2 & 136,346 & 133,6 & 134,1 & 134,3 & 134,79 \\
\hline
\end{tabular}

Errores en unidades: en la siguiente que tuvieron los pronósticos de cada uno de tabla se pueden ver los errores en unidades los métodos frente al dato histórico.

\begin{tabular}{|c|c|c|c|c|c|c|c|c|c|c|c|}
\hline \multirow{2}{*}{ Per } & \multirow{2}{*}{$\begin{array}{c}\text { Demanda } \\
\text { real }\end{array}$} & \multicolumn{3}{|c|}{ Media Móvil } & \multirow{2}{*}{$\begin{array}{c}\text { Método } \\
\text { de } \\
\text { Brown }\end{array}$} & \multirow{2}{*}{$\begin{array}{c}\text { Método } \\
\text { de } \\
\text { Winters }\end{array}$} & \multirow{2}{*}{$\begin{array}{l}\text { Método } \\
\text { de } \\
\text { Holt }\end{array}$} & \multicolumn{3}{|c|}{ Suavización exponencial simple } & \multirow{2}{*}{$\begin{array}{c}\text { Método } \\
\text { Torres }\end{array}$} \\
\hline & & $n=12$ & $n=6$ & $n=3$ & & & & Alpha $=0,5$ & Alpha $=0,7$ & Alpha $=0,3$ & \\
\hline Ene & 129 & 1,900 & 1,300 & 0,700 & 4,320 & 0,082 & 4,251 & 3,400 & 4,000 & 4,100 & 2,400 \\
\hline Feb & 134 & 3,300 & 4,000 & 4,400 & 0,766 & 0,928 & 0,831 & 1,800 & 1,300 & 1,100 & 4,600 \\
\hline Mar & 135 & 4,300 & 5,700 & 5,300 & 1,752 & 2,662 & 1,813 & 3,000 & 2,400 & 2,300 & 1,080 \\
\hline Abr & 133 & 2,000 & 3,100 & 2,400 & 0,862 & 0,954 & 0,805 & 0,500 & 0,000 & 0,100 & 2,210 \\
\hline May & 133 & 2,300 & 3,200 & 2,900 & 0,976 & 0,515 & 0,922 & 0,600 & 0,100 & 0,100 & 0,010 \\
\hline Jun & 134 & 2,500 & 3,500 & 4,400 & 0,890 & 1,947 & 0,840 & 0,800 & 0,300 & 0,200 & 0,840 \\
\hline Jul & 132 & 0,600 & 1,300 & 2,500 & 3,004 & 0,611 & 2,958 & 1,100 & 1,600 & 1,800 & 0,670 \\
\hline Ago & 135 & 3,300 & 4,100 & 5,400 & 0,018 & 0,995 & 0,025 & 2,100 & 1,500 & 1,400 & 2,330 \\
\hline Sep & 134 & 1,300 & 1,700 & 2,500 & 1,932 & 0,902 & 1,893 & 0,300 & 0,200 & 0,300 & 1,100 \\
\hline Oct & 134 & 1,400 & 1,600 & 2,100 & 1,946 & 1,182 & 1,911 & 0,500 & 0,100 & 0,200 & 0,480 \\
\hline Nov & 135 & 2,500 & 2,700 & 2,800 & 0,960 & 0,819 & 0,929 & 1,600 & 1,100 & 1,000 & 1,250 \\
\hline Dic & 135 & 1,900 & 2,300 & 2,300 & 1,474 & 0,271 & 1,446 & 1,300 & 0,800 & 0,600 & 0,110 \\
\hline
\end{tabular}

Con este método de prueba se mide el menor error cuadrático partiendo de la diferencia entre el dato pronosticado menos la demanda y ese resultado elevado al cuadrado. Con la suma de todos los errores al cuadrado RSE se puede determinar cuál estuvo más cerca del dato real. Los valores de las diferencias al cuadrado se muestran a continuación: 


\begin{tabular}{|c|c|c|c|c|c|c|c|c|c|c|c|}
\hline \multirow{2}{*}{ Per } & \multirow{2}{*}{$\begin{array}{l}\text { Demanda } \\
\text { real }\end{array}$} & \multicolumn{3}{|c|}{ Media Móvil } & \multirow{2}{*}{$\begin{array}{l}\text { Método } \\
\text { de } \\
\text { Brown }\end{array}$} & \multirow{2}{*}{$\begin{array}{c}\text { Método } \\
\text { de } \\
\text { Winters }\end{array}$} & \multirow{2}{*}{$\begin{array}{c}\text { Método } \\
\text { de } \\
\text { Holt }\end{array}$} & \multicolumn{3}{|c|}{ Suavización exponencial simple } & \multirow{2}{*}{$\begin{array}{l}\text { Método } \\
\text { Torres }\end{array}$} \\
\hline & & $\mathrm{n}=12$ & $n=6$ & $\mathbf{n}=\mathbf{3}$ & & & & Alpha $=0,5$ & Alpha $=0,7$ & Alpha $=0,3$ & \\
\hline Ene & 129 & 3,610 & 1,690 & 0,490 & 18,662 & 0,007 & 18,071 & 11,560 & 16,000 & 16,810 & 5,760 \\
\hline Feb & 134 & 10,890 & 16,000 & 19,360 & 0,587 & 0,861 & 0,691 & 3,240 & 1,690 & 1,210 & 21,160 \\
\hline Mar & 135 & 18,490 & 32,490 & 28,090 & 3,070 & 7,084 & 3,287 & 9,000 & 5,760 & 5,290 & 1,166 \\
\hline Abr & 133 & 4,000 & 9,610 & 5,760 & 0,743 & 0,910 & 0,648 & 0,250 & 0,000 & 0,010 & 4,884 \\
\hline May & 133 & 5,290 & 10,240 & 8,410 & 0,953 & 0,265 & 0,850 & 0,360 & 0,010 & 0,010 & 0,000 \\
\hline Jun & 134 & 6,250 & 12,250 & 19,360 & 0,792 & 3,790 & 0,706 & 0,640 & 0,090 & 0,040 & 0,706 \\
\hline Jul & 132 & 0,360 & 1,690 & 6,250 & 9,024 & 0,373 & 8,750 & 1,210 & 2,560 & 3,240 & 0,449 \\
\hline Ago & 135 & 10,890 & 16,810 & 29,160 & 0,000 & 0,990 & 0,001 & 4,410 & 2,250 & 1,960 & 5,429 \\
\hline Sep & 134 & 1,690 & 2,890 & 6,250 & 3,733 & 0,813 & 3,583 & 0,090 & 0,040 & 0,090 & 1,210 \\
\hline Oct & 134 & 1,960 & 2,560 & 4,410 & 3,787 & 1,397 & 3,652 & 0,250 & 0,010 & 0,040 & 0,230 \\
\hline Nov & 135 & 6,250 & 7,290 & 7,840 & 0,922 & 0,672 & 0,863 & 2,560 & 1,210 & 1,000 & 1,563 \\
\hline Dic & 135 & 3,610 & 5,290 & 5,290 & 2,173 & 0,073 & 2,091 & 1,690 & 0,640 & 0,360 & 0,012 \\
\hline \multicolumn{2}{|c|}{ Total } & 73,290 & 118,810 & 140,670 & 44,445 & 17,236 & 43,192 & 35,260 & 30,260 & 30,060 & 42,569 \\
\hline
\end{tabular}

Los resultados de la clasificación son los siguientes:

\begin{tabular}{|l|l|c|c||}
\hline \hline Ranking & \multicolumn{1}{|c|}{ Método } & $\begin{array}{c}\text { Suma Errores } \\
\text { cuadrado }\end{array}$ & $\begin{array}{c}\text { Desviación } \\
\text { Porcentual }\end{array}$ \\
\hline Primero & Winters & 17,236 & $0,00 \%$ \\
\hline Segundo & Suavización Alpha 0,7 & 30,060 & $74,40 \%$ \\
\hline Tercero & Suavización Alpha 0,5 & 30,260 & $75,56 \%$ \\
\hline Cuarto & Suavización Alpha 0,3 & 35,260 & $104,57 \%$ \\
\hline Quinto & Torres & $\mathbf{4 2 , 5 6 9}$ & $\mathbf{1 4 6 , 9 8 \%}$ \\
\hline Sexto & Holt & 43,192 & $150,59 \%$ \\
\hline Séptimo & Brown & 44,445 & $157,86 \%$ \\
\hline Octavo & Promedio Móvil N = 12 & 73,290 & $325,21 \%$ \\
\hline Noveno & Promedio Móvil N = 6 & 118,810 & $589,31 \%$ \\
\hline Décimo & Promedio Móvil N = 3 & 140,670 & $716,14 \%$ \\
\hline
\end{tabular}

PRUEBA NÚMERO TRES

Para realizar una tercera prueba se eligió una nueva serie de datos que representa el consumo promedio de Barriles ${ }^{11}$ de gasolina en Colombia en los últimos seis años es decir, una serie de setenta y dos datos. Los últimos doce datos se reservan para probar la eficiencia de los métodos usados. A continuación pueden verse los datos originales: 


\begin{tabular}{||l|c|c|c|c|c|c||}
\hline Meses & Año 1 & Año 2 & Año 3 & Año 4 & Año 5 & Año 6 \\
\hline ENERO & 4.051 .669 & 3.428 .972 & 3.465 .862 & 2.919 .208 & 2.835 .900 & 2.926 .130 \\
\hline FEBRERO & 3.583 .328 & 3.523 .296 & 3.120 .432 & 2.820 .748 & 2.540 .126 & 2.631 .980 \\
\hline MARZO & 3.957 .212 & 3.916 .447 & 3.411 .364 & 2.831 .137 & 2.733 .109 & 2.751 .183 \\
\hline ABRIL & 4.041 .930 & 3.493 .440 & 2.960 .790 & 2.773 .560 & 2.615 .014 & 2.505 .301 \\
\hline MAYO & 3.923 .608 & 3.666 .091 & 2.995 .530 & 2.846 .079 & 2.701 .131 & 2.704 .137 \\
\hline JUNIO & 3.783 .510 & 2.925 .570 & 3.151 .680 & 2.783 .370 & 2.602 .845 & 2.633 .125 \\
\hline JULIO & 4.068 .037 & 3.457 .771 & 2.836 .531 & 2.765 .355 & 2.754 .818 & 2.616 .546 \\
\hline AGOSTO & 3.909 .565 & 3.657 .721 & 3.299 .919 & 2.860 .773 & 2.849 .311 & 2.679 .817 \\
\hline SEPTIEMBRE & 3.882 .690 & 3.443 .310 & 2.987 .790 & 2.652 .210 & 2.693 .766 & 2.549 .386 \\
\hline OCTUBRE & 4.045 .035 & 3.339 .444 & 3.047 .796 & 2.779 .336 & 2.854 .967 & 2.680 .441 \\
\hline NOVIEMBRE & 3.616 .980 & 3.646 .680 & 2.847 .810 & 2.770 .260 & 2.766 .433 & 2.601 .652 \\
\hline DICIEMBRE & 4.387 .182 & 3.536 .945 & 3.360 .307 & 2.868 .585 & 3.070 .043 & 3.063 .701 \\
\hline
\end{tabular}

En la siguiente gráfica se puede observar de gasolina (medido en barriles al mes), el comportamiento del consumo nacional desde el año 1998 hasta el año 2003.

\section{Consumo nacional de combustibles}

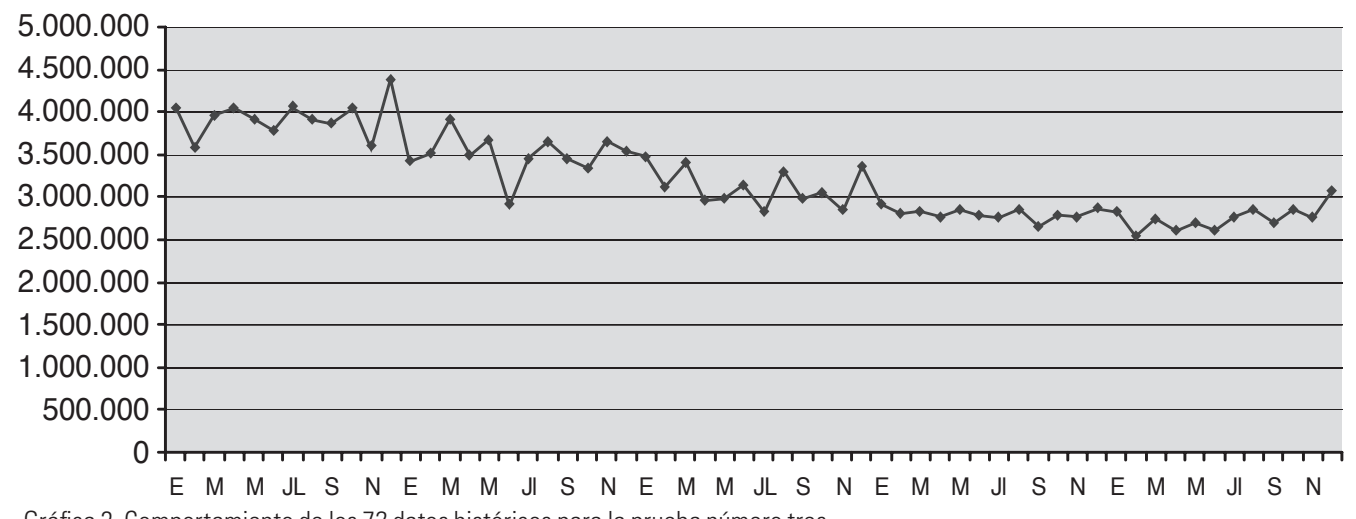

Gráfica 3. Comportamiento de los 72 datos históricos para la prueba número tres

Los doce datos reservados para hacer la comparación son los siguientes:

\begin{tabular}{|l|c|l|c|}
\hline \multicolumn{1}{|c|}{ Mes } & \multicolumn{1}{c|}{ Año 9 } & \multicolumn{1}{c|}{ Mes } & \multicolumn{1}{c|}{ Año 9 } \\
\hline Enero & 2.926 .130 & Julio & 2.616 .546 \\
\hline Febrero & 2.631 .980 & Agosto & 2.679 .817 \\
\hline Marzo & 2.751 .183 & Septiembre & 2.549 .386 \\
\hline Abril & 2.505 .301 & Octubre & 2.680 .441 \\
\hline Mayo & 2.704 .137 & Noviembre & 2.601 .652 \\
\hline Junio & 2.633 .125 & Diciembre & 3.063 .701 \\
\hline
\end{tabular}

En las siguientes pantallas se muestran los resultados de la ejecución del programa Statgraphics ${ }^{\circledR}$ junto a los valores de los índices $\alpha, \gamma$ y $\beta$ determinados automáticamente con la ayuda de este software: 


\section{Gráfico de Secuencia de tiempo para Gasolina}

(X 100000)

Suavizado exp. lineal de Holt con alfa $=0,1395$ y beta $=0,1475$

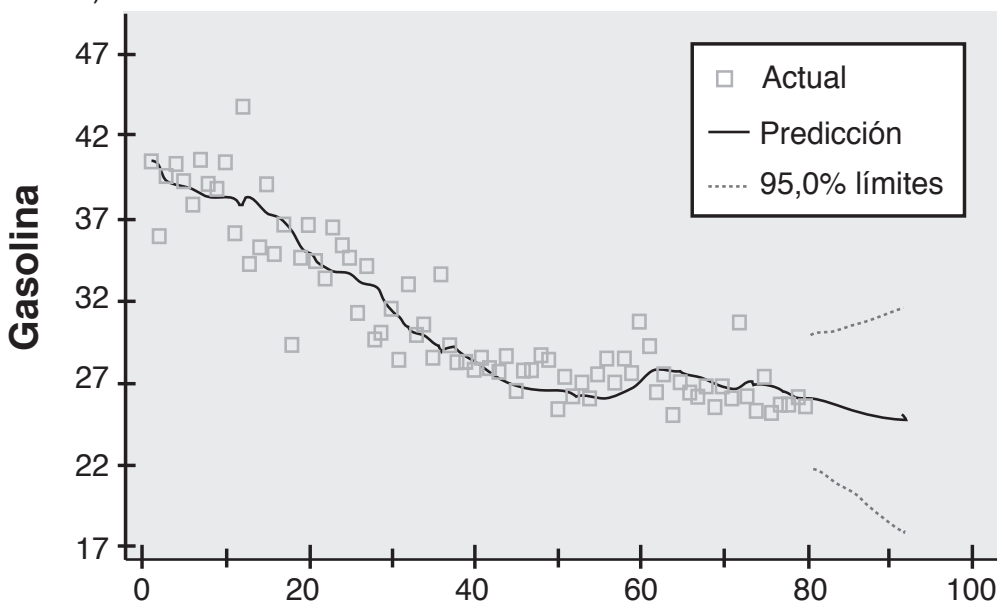

Gráfico de Secuencia de tiempo para Gasolina

(X 100000) Suavizado exp. lineal de Brown con alfa $=0,1608$

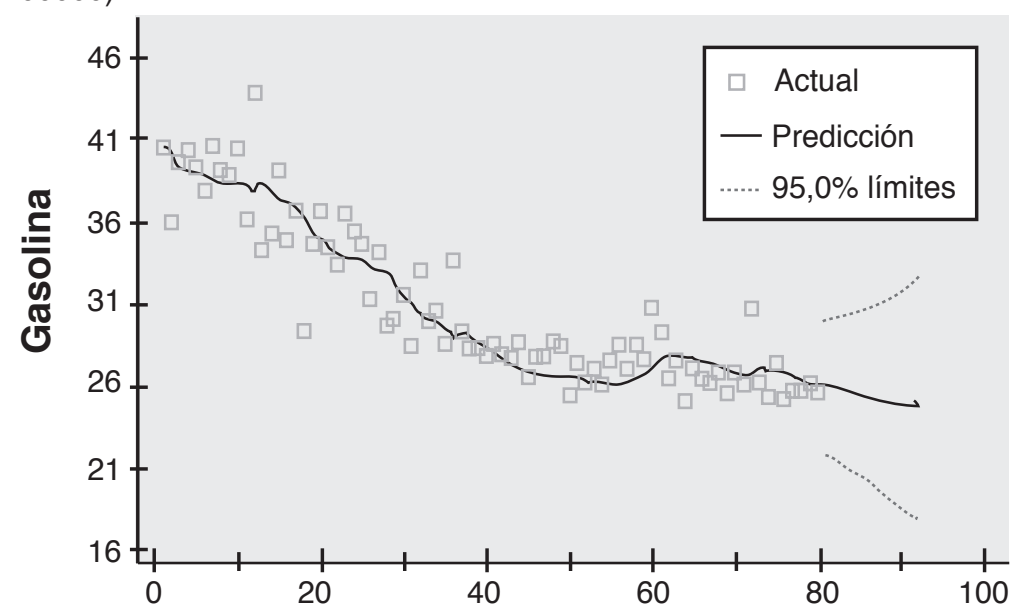


Los resultados de los pronósticos usando cada uno de los métodos son los siguientes:

\begin{tabular}{|c|c|c|c|c|c|c|}
\hline \multirow{2}{*}{ Períodos } & \multirow{2}{*}{$\begin{array}{l}\text { Demanda } \\
\text { real }\end{array}$} & \multicolumn{3}{|c|}{ Media Móvil } & \multirow{2}{*}{$\begin{array}{l}\text { Método de } \\
\text { Brown }\end{array}$} & \multirow{2}{*}{$\begin{array}{l}\text { Método de } \\
\text { Winters }\end{array}$} \\
\hline & & $n=12$ & $n=6$ & $n=3$ & & \\
\hline Ene & 2.926 .130 & 2750243 & 2640378 & 2651269 & 2630260 & 2.478 .473 \\
\hline Feb & 2.631 .980 & 2741987 & 2641778 & 2633646 & 2620080 & 2.564 .734 \\
\hline Mar & 2.751 .183 & 2746006 & 2623544 & 2633820 & 2609890 & 2.477 .378 \\
\hline Abr & 2.505 .301 & 2736926 & 2643251 & 2639579 & 2599700 & 2.787 .369 \\
\hline May & 2.704 .137 & 2734467 & 2633104 & 2635682 & 2589520 & 2.524 .434 \\
\hline Jun & 2.633 .125 & 2706502 & 2633100 & 2636360 & 2579330 & 2.362 .485 \\
\hline Jul & 2.616 .546 & 2688200 & 2635859 & 2637207 & 2569140 & 2.521 .111 \\
\hline Ago & 2.679 .817 & 2692885 & 2635106 & 2636416 & 2558960 & 2.321 .650 \\
\hline Sep & 2.549 .386 & 2688027 & 2633994 & 2636661 & 2548770 & 2.425 .739 \\
\hline Oct & 2.680 .441 & 2703254 & 2635736 & 2636761 & 2538580 & 2.351 .006 \\
\hline Nov & 2.601 .652 & 2703181 & 2634483 & 2636613 & 2528400 & 2.380 .815 \\
\hline Dic & 3.063.701 & 2709019 & 2634713 & 2636679 & 2518210 & 2.464 .163 \\
\hline
\end{tabular}

\begin{tabular}{|c|c|c|c|c|c|c|}
\hline \multirow[b]{2}{*}{ Períodos } & \multirow{2}{*}{$\begin{array}{l}\text { Demanda } \\
\text { real }\end{array}$} & \multirow{2}{*}{$\begin{array}{l}\text { Método de } \\
\text { Holt }\end{array}$} & \multicolumn{3}{|c|}{ Suavización exponencial simple } & \multirow{2}{*}{$\begin{array}{l}\text { Método } \\
\text { Torres }\end{array}$} \\
\hline & & & Alpha $=0,3$ & Alpha $=0,5$ & Alpha $=0,7$ & \\
\hline Ene & 2.926 .130 & 2650060 & 2642189 & 2613489 & 2603995 & 2838652 \\
\hline Feb & 2.631 .980 & 2648450 & 2621714 & 2593014 & 2583520 & 2452023 \\
\hline Mar & 2.751 .183 & 2646840 & 2601238 & 2572539 & 2563044 & 2892366 \\
\hline Abr & 2.505 .301 & 2645230 & 2580763 & 2552063 & 2542569 & 2491402 \\
\hline May & 2.704 .137 & 2643620 & 2560288 & 2531588 & 2522093 & 2914986 \\
\hline Jun & 2.633 .125 & 2642010 & 2539812 & 2511112 & 2501618 & 2569408 \\
\hline Jul & 2.616 .546 & 2640400 & 2519337 & 2490637 & 2481143 & 2660738 \\
\hline Ago & 2.679 .817 & 2638800 & 2498861 & 2470161 & 2460667 & 2732878 \\
\hline Sep & 2.549 .386 & 2637190 & 2478386 & 2449686 & 2440192 & 2496049 \\
\hline Oct & 2.680 .441 & 2635580 & 2457910 & 2429211 & 2419716 & 2822310 \\
\hline Nov & 2.601 .652 & 2633970 & 2437435 & 2408735 & 2399241 & 2536239 \\
\hline Dic & 3.063.701 & 2632360 & 2416960 & 2388260 & 2378765 & 3134661 \\
\hline
\end{tabular}


Errores en unidades: en la siguiente que tuvieron los pronósticos de cada uno de tabla se pueden ver los errores en unidades los métodos frente al dato histórico.

\begin{tabular}{|c|c|c|c|c|c|c|}
\hline \multirow{2}{*}{ Períodos } & \multirow{2}{*}{$\begin{array}{l}\text { Demanda } \\
\text { real }\end{array}$} & \multicolumn{3}{|c|}{ Media Móvil } & \multirow{2}{*}{$\begin{array}{l}\text { Método de } \\
\text { Brown }\end{array}$} & \multirow{2}{*}{$\begin{array}{l}\text { Método de } \\
\text { Winters }\end{array}$} \\
\hline & & $n=12$ & $\mathrm{n}=6$ & $\mathrm{n}=3$ & & \\
\hline Ene & 2.926 .130 & 175.887 & 285.752 & 274.861 & 295.870 & 447.657 \\
\hline Feb & 2.631 .980 & 110.007 & 9.798 & 1.666 & 11.900 & 67.246 \\
\hline Mar & 2.751 .183 & 5.177 & 127.639 & 117.363 & 141.293 & 273.805 \\
\hline Abr & 2.505 .301 & 231.625 & 137.950 & 134.278 & 94.399 & 282.068 \\
\hline May & 2.704 .137 & 30.330 & 71.033 & 68.455 & 114.617 & 179.703 \\
\hline Jun & 2.633 .125 & 73.377 & 25 & 3.235 & 53.795 & 270.640 \\
\hline Jul & 2.616 .546 & 71.654 & 19.313 & 20.661 & 47.406 & 95.435 \\
\hline Ago & 2.679 .817 & 13.068 & 44.711 & 43.401 & 120.857 & 358.167 \\
\hline Sep & 2.549 .386 & 138.641 & 84.608 & 87.275 & 616 & 123.647 \\
\hline Oct & 2.680 .441 & 22.813 & 44.705 & 43.680 & 141.861 & 329.435 \\
\hline Nov & 2.601 .652 & 101.529 & 32.831 & 34.961 & 73.252 & 220.837 \\
\hline Dic & 3.063 .701 & 354.682 & 428.988 & 427.022 & 545.491 & 599.538 \\
\hline
\end{tabular}

\begin{tabular}{|c|c|c|c|c|c|c|}
\hline \multirow{2}{*}{ Períodos } & \multirow{2}{*}{$\begin{array}{l}\text { Demanda } \\
\text { real }\end{array}$} & \multirow{2}{*}{$\begin{array}{l}\text { Método de } \\
\text { Holt }\end{array}$} & \multicolumn{3}{|c|}{ Suavización exponencial simple } & \multirow{2}{*}{$\begin{array}{l}\text { Método } \\
\text { Torres }\end{array}$} \\
\hline & & & Alpha $=0,3$ & Alpha $=0,5$ & Alpha $=0,7$ & \\
\hline Ene & 2.926 .130 & 276.070 & 283.941 & 312.641 & 322.135 & 87.478 \\
\hline Feb & 2.631 .980 & 16.470 & 10.266 & 38.966 & 48.460 & 179.957 \\
\hline Mar & 2.751 .183 & 104.343 & 149.945 & 178.644 & 188.139 & 141.183 \\
\hline Abr & 2.505 .301 & 139.929 & 75.462 & 46.762 & 37.268 & 13.899 \\
\hline May & 2.704 .137 & 60.517 & 143.849 & 172.549 & 182.044 & 210.849 \\
\hline Jun & 2.633 .125 & 8.885 & 93.313 & 122.013 & 131.507 & 63.717 \\
\hline Jul & 2.616 .546 & 23.854 & 97.209 & 125.909 & 135.403 & 44.192 \\
\hline Ago & 2.679 .817 & 41.017 & 180.956 & 209.656 & 219.150 & 53.061 \\
\hline Sep & 2.549 .386 & 87.804 & 71.000 & 99.700 & 109.194 & 53.337 \\
\hline Oct & 2.680 .441 & 44.861 & 222.531 & 251.230 & 260.725 & 141.869 \\
\hline Nov & 2.601 .652 & 32.318 & 164.217 & 192.917 & 202.411 & 65.413 \\
\hline Dic & 3.063 .701 & 431.341 & 646.741 & 675.441 & 684.936 & 70.960 \\
\hline
\end{tabular}

Con este método de prueba se mide el menor error cuadrático partiendo de la diferencia entre el dato pronosticado menos la demanda y ese resultado elevado al cuadrado. Con la suma de todos los errores al cuadrado RSE se puede determinar cuál estuvo más cerca del dato real. Los valores de las diferencias al cuadrado se muestran en las siguientes tablas: 


\begin{tabular}{|c|c|c|c|c|c|}
\hline \multirow{2}{*}{$\begin{array}{c}\text { Demanda } \\
\text { real }\end{array}$} & \multicolumn{3}{|c|}{ Media Móvil } & \multirow{2}{*}{ Método de Brown } & \multirow{2}{*}{ Método de Winters } \\
\hline & $n=12$ & $\mathrm{n}=6$ & $n=3$ & & \\
\hline 2.926 .130 & 30.936 .295 .398 & 81.654 .110 .253 & 75.548 .569 .321 & 87.539 .056 .900 & 200.397 .174 .634 \\
\hline 2.631 .980 & 12.101 .591 .997 & 96.001 .348 & 2.776 .667 & 141.610 .000 & 4.522.083.693 \\
\hline 2.751 .183 & 26.803 .858 & 16.291 .706 .048 & 13.773.969.447 & 19.963 .711 .849 & 74.969.427.188 \\
\hline 2.505 .301 & 53.649 .979 .462 & 19.030 .258 .915 & 18.030 .471 .872 & 8.911 .171 .201 & 79.562 .232 .514 \\
\hline 2.704 .137 & 919.896 .148 & 5.045 .747 .909 & 4.686.115.759 & 13.137.056.689 & 32.293.178.272 \\
\hline 2.633 .125 & 5.384 .211 .855 & 617 & 10.467 .009 & 2.893.902.025 & 73.246.188.223 \\
\hline 2.616 .546 & 5.134 .289 .220 & 372.999 .504 & 426.872 .216 & 2.247 .328 .836 & 9.107 .828 .727 \\
\hline 2.679 .817 & 170.773 .519 & 1.999 .070 .558 & 1.883 .619 .256 & 14.606 .414 .449 & 128.283 .786 .136 \\
\hline 2.549 .386 & 19.221 .314 .055 & 7.158.519.422 & 7.616.953.506 & 379.456 & 15.288 .689 .913 \\
\hline 2.680 .441 & 520.438 .287 & 1.998.563.762 & 1.907 .902 .704 & 20.124 .543 .321 & 108.527 .469 .958 \\
\hline 2.601 .652 & 10.308 .045 .031 & 1.077 .882 .247 & 1.222 .269 .924 & 5.365 .855 .504 & 48.769.177.997 \\
\hline 3.063 .701 & 125.799.613.254 & 184.030 .668 .759 & 182.348.189.518 & 297.560 .431 .081 & 359.445 .489 .694 \\
\hline TOTALES & 264.173 .252 .082 & 318.755 .529 .342 & 307.458 .177 .199 & 472.491 .461 .311 & 1.134 .412 .726 .949 \\
\hline
\end{tabular}

\begin{tabular}{|c|c|c|c|c|c|}
\hline \multirow{2}{*}{$\begin{array}{l}\text { Demanda } \\
\text { real }\end{array}$} & \multirow{2}{*}{ Método de Holt } & \multicolumn{3}{|c|}{ Suavización exponencial simple } & \multirow{2}{*}{ Método Torres } \\
\hline & & Alpha $=0,3$ & Alpha $=0,5$ & Alpha $=0,7$ & \\
\hline 2.926 .130 & 76.214 .644 .900 & 80.622 .491 .481 & 97.744 .394 .881 & 103.770.958.225 & 7.652 .400 .484 \\
\hline 2.631 .980 & 271.260 .900 & 105.390 .756 & 1.518 .349 .156 & 2.348 .371 .600 & 32.384 .521 .849 \\
\hline 2.751 .183 & 10.887 .461 .649 & 22.483 .503 .025 & 31.913 .678 .736 & 35.396 .283 .321 & 19.932 .639 .489 \\
\hline 2.505 .301 & 19.580 .125 .041 & 5.694 .513 .444 & 2.186 .684 .644 & 1.388 .903 .824 & 193.182.201 \\
\hline 2.704 .137 & 3.662.307.289 & 20.692 .534 .801 & 29.773.157.401 & 33.140 .017 .936 & 44.457 .300 .801 \\
\hline 2.633 .125 & 78.943 .225 & 8.707 .315 .969 & 14.887.172.169 & 17.294 .091 .049 & 4.059 .856 .089 \\
\hline 2.616 .546 & 569.013 .316 & 9.449 .589 .681 & 15.853 .076 .281 & 18.333 .972 .409 & 1.952 .932 .864 \\
\hline 2.679 .817 & 1.682 .394 .289 & 32.745 .073 .936 & 43.955 .638 .336 & 48.026 .722 .500 & 2.815 .469 .721 \\
\hline 2.549 .386 & 7.709 .542 .416 & 5.041 .000 .000 & 9.940 .090 .000 & 11.923 .329 .636 & 2.844 .835 .569 \\
\hline 2.680 .441 & 2.012 .509 .321 & 49.520 .045 .961 & 63.116 .512 .900 & 67.977 .525 .625 & 20.126.813.161 \\
\hline 2.601 .652 & 1.044 .453 .124 & 26.967.223.089 & 37.216 .968 .889 & 40.970 .212 .921 & 4.278 .860 .569 \\
\hline 3.063 .701 & 186.055 .058 .281 & 418.273 .921 .081 & 456.220 .544 .481 & 469.137.324.096 & 5.035 .321 .600 \\
\hline TOTALES & 309.767 .713 .751 & 680.302 .603 .224 & 804.326 .267 .874 & 849.707 .713 .142 & 145.734 .134 .397 \\
\hline
\end{tabular}


Los resultados de clasificación son los siguientes:

\begin{tabular}{|l|l|r|r|}
\hline Ranking & \multicolumn{1}{|c|}{ Método } & \multicolumn{1}{|c|}{$\begin{array}{c}\text { Suma Errores } \\
\text { cuadrado }\end{array}$} & $\begin{array}{r}\text { Desviación } \\
\text { Porcentual }\end{array}$ \\
\hline Primero & Torres & $\mathbf{1 4 5 . 7 3 4 . 1 3 4 . 3 9 7}$ & $\mathbf{0 , 0 0 \%}$ \\
\hline Segundo & Promedio Móvil N = 12 & 264.173 .252 .082 & $81,27 \%$ \\
\hline Tercero & Promedio Móvil N = 3 & 307.458 .177 .199 & $110,97 \%$ \\
\hline Cuarto & Holt & 309.767 .713 .751 & $112,56 \%$ \\
\hline Quinto & Promedio Móvil N = 6 & 318.755 .529 .342 & $118,72 \%$ \\
\hline Sexto & Brown & 472.491 .461 .311 & $224,21 \%$ \\
\hline Séptimo & Suavización Alpha 0,3 & 680.302 .603 .224 & $366,81 \%$ \\
\hline Octavo & Suavización Alpha 0,5 & 804.326 .267 .874 & $451,91 \%$ \\
\hline Noveno & Suavización Alpha 0,7 & 849.707 .713 .142 & $483,05 \%$ \\
\hline Décimo & Winters & 1.134 .412 .726 .949 & $678,41 \%$ \\
\hline \hline
\end{tabular}

PRUEBA NÚMERO CUATRO

Para realizar una cuarta prueba se eligió una nueva serie de datos que representa el consumo promedio de papel ${ }^{12}$ en Colombia dado en miles de toneladas de papel para el consumo interno desde el año 1974 hasta el año 2003. Es decir, una serie de treinta datos. Los últimos cinco datos se reservan para probar la eficiencia de los métodos usados. En la siguiente tabla pueden verse los datos originales:

\begin{tabular}{||c|c|c|c||}
\hline Año & Producción & Año & Producción \\
\hline 1974 & 289 & 1989 & 511 \\
\hline 1975 & 248 & 1990 & 534 \\
\hline 1976 & 287 & 1991 & 563 \\
\hline 1977 & 295 & 1992 & 627 \\
\hline 1978 & 321 & 1993 & 610 \\
\hline 1979 & 342 & 1994 & 673 \\
\hline 1980 & 367 & 1995 & 677 \\
\hline 1981 & 372 & 1996 & 691 \\
\hline 1982 & 366 & 1997 & 705 \\
\hline 1983 & 365 & 1998 & 711 \\
\hline 1984 & 412 & 1999 & 734 \\
\hline 1985 & 446 & 2000 & 771 \\
\hline 1986 & 457 & 2001 & 772 \\
\hline 1987 & 488 & 2002 & 848 \\
\hline 1988 & 504 & 2003 & 865 \\
\hline \hline
\end{tabular}

En la siguiente gráfica se puede observar el comportamiento del consumo nacional de papel (medido en miles de toneladas al año), desde el año 1974 hasta el año 2003. Se tomarán las demandas de 1994 hasta 1998 para pronosticar las demandas de 1999 hasta el 2003. La información real de estos años se reservará para medir la eficiencia del método de Torres. 


\section{Producción de papel}

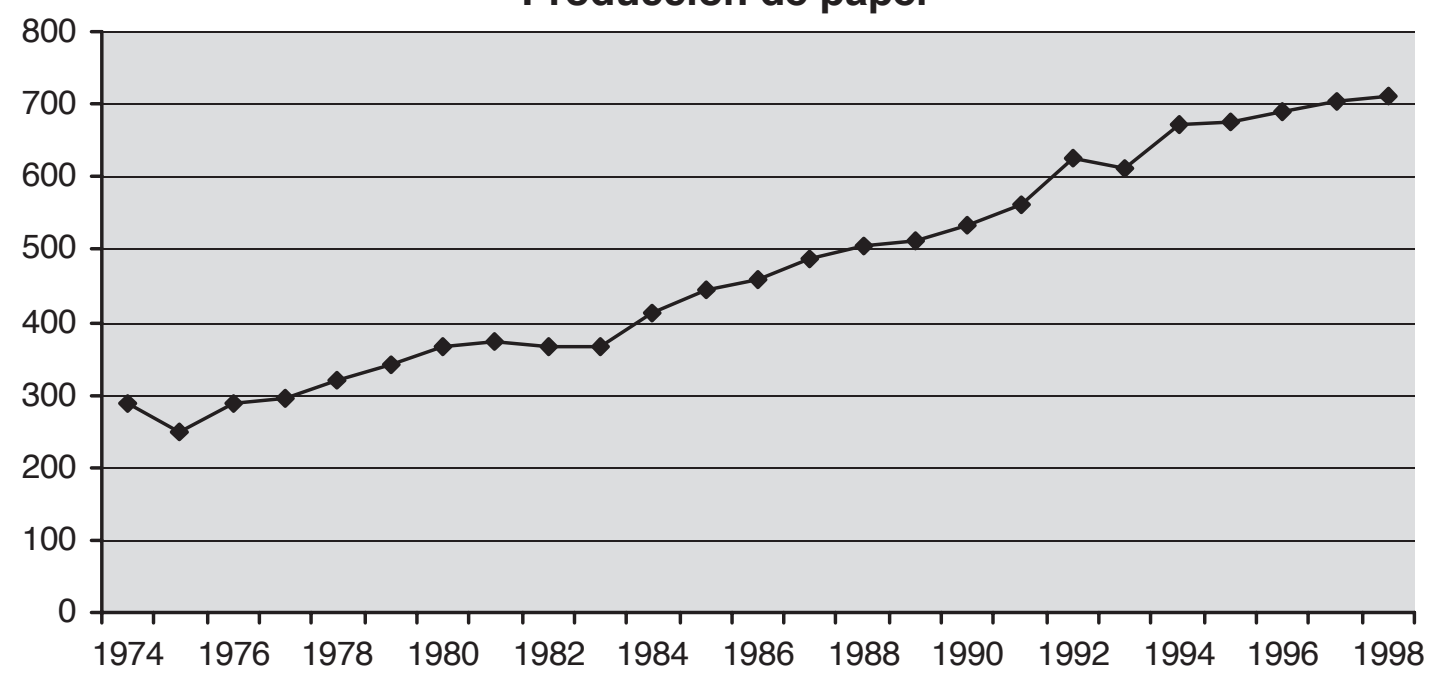

Gráfica 4. Comportamiento de los 30 datos históricos para la prueba número cuatro.

Los cinco datos reservados para hacer la comparación son los siguientes:

\begin{tabular}{||c|c|}
\hline Año & Producción \\
\hline 1999 & 734 \\
\hline 2000 & 771 \\
\hline 2001 & 772 \\
\hline 2002 & 848 \\
\hline 2003 & 865 \\
\hline
\end{tabular}

En las siguientes pantallas se muestran los resultados de la ejecución del programa Statgraphics ${ }^{\circledR}$ junto a los valores de los índices $\alpha, \gamma$ y $\beta$ determinados automáticamente con la ayuda de este software:

\section{Gráfico de Secuencia de tiempo para Papel}

Suavizado exp. lineal de Holt con alfa $=0,8876$ y beta $=0,0822$

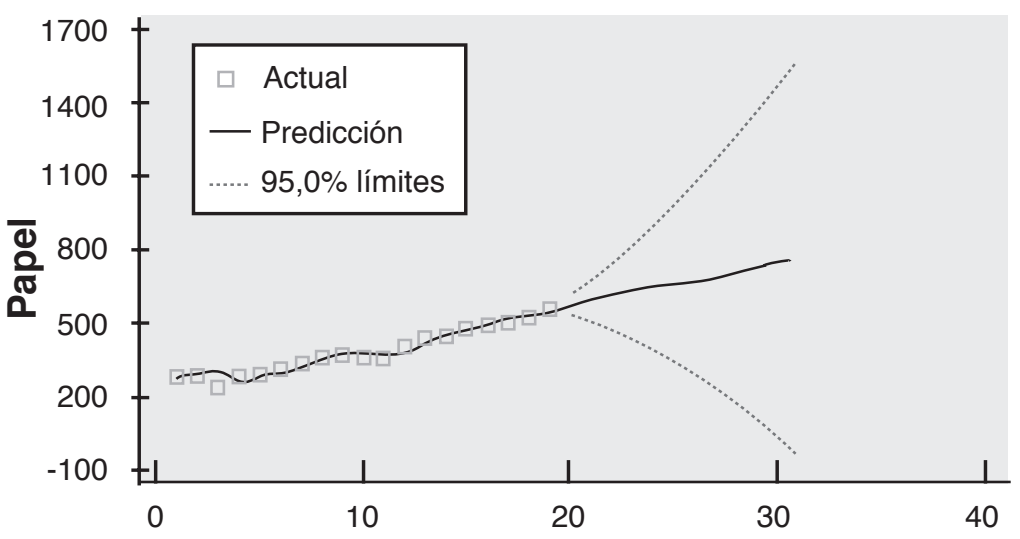




\section{Gráfico de Secuencia de tiempo para Papel}

Suavizado exp. lineal de Brown con alfa $=0,5023$

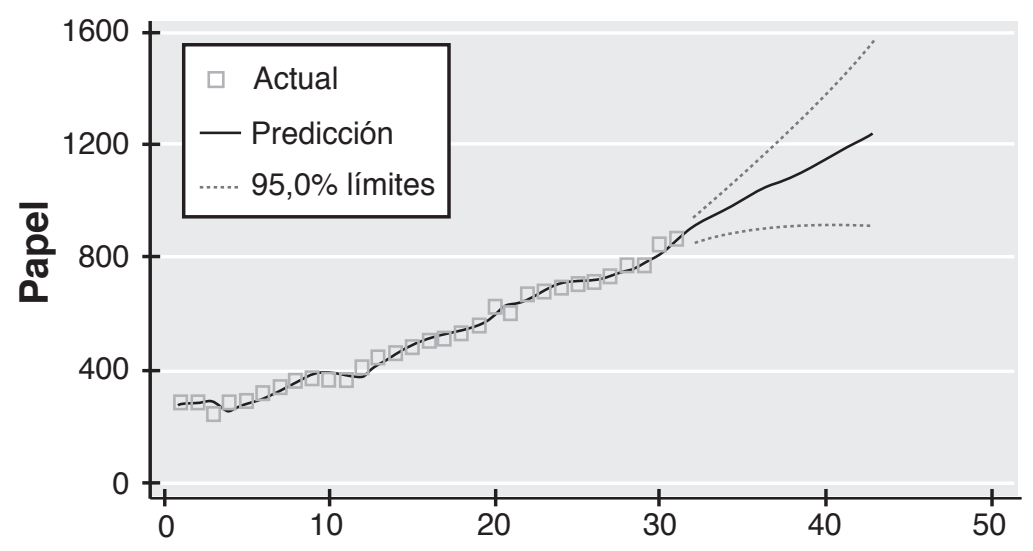

Los resultados de los pronósticos usando cada uno de los métodos son los siguientes:

\begin{tabular}{|c|c|c|c|c|c|c|c|c|c|c|c|}
\hline \multirow{2}{*}{ Año } & \multirow{2}{*}{$\underset{\text { real }}{\text { Demanda }}$} & \multicolumn{3}{|c|}{ Media Móvil } & \multirow{2}{*}{$\begin{array}{c}\text { Método } \\
\text { de } \\
\text { Brown }\end{array}$} & \multirow{2}{*}{$\begin{array}{c}\text { Método } \\
\text { de } \\
\text { Winters }\end{array}$} & \multirow{2}{*}{$\begin{array}{l}\text { Método } \\
\text { de Holt }\end{array}$} & \multicolumn{3}{|c|}{ Suavización exponencial simple } & \multirow{2}{*}{$\begin{array}{l}\text { Método } \\
\text { Torres }\end{array}$} \\
\hline & & $n=12$ & $\mathrm{n}=6$ & $n=3$ & & & & Alpha $=0,3$ & Alpha $=0,5$ & Alpha $=0,7$ & \\
\hline 1999 & 734 & 449 & 509 & 536 & 569 & 598 & 570 & 527 & 529 & 528 & 678 \\
\hline 2000 & 770 & 455 & 518 & 544 & 587 & 612 & 590 & 543 & 544 & 543 & 684 \\
\hline 2001 & 772 & 462 & 523 & 548 & 605 & 623 & 610 & 558 & 560 & 559 & 692 \\
\hline 2002 & 847 & 471 & 526 & 543 & 623 & 659 & 631 & 574 & 575 & 574 & 710 \\
\hline 2003 & 865 & 479 & 529 & 545 & 641 & 599 & 651 & 589 & 591 & 589 & 715 \\
\hline
\end{tabular}

Errores en unidades: en la siguiente que tuvieron los pronósticos de cada uno tabla se pueden ver los errores en unidades de los métodos frente al dato histórico.

\begin{tabular}{|c|c|c|c|c|c|c|c|c|c|c|c|}
\hline \multirow{2}{*}{ Año } & \multirow{2}{*}{$\begin{array}{c}\text { Demanda } \\
\text { real }\end{array}$} & \multicolumn{3}{|c|}{ Media Móvil } & \multirow{2}{*}{$\begin{array}{c}\text { Método } \\
\text { de } \\
\text { Brown }\end{array}$} & \multirow{2}{*}{$\begin{array}{l}\text { Método } \\
\text { de } \\
\text { Winters }\end{array}$} & \multirow{2}{*}{$\begin{array}{c}\text { Método } \\
\text { de } \\
\text { Holt }\end{array}$} & \multicolumn{3}{|c|}{ Suavización exponencial simple } & \multirow{2}{*}{$\begin{array}{l}\text { Método } \\
\text { Torres }\end{array}$} \\
\hline & & $n=12$ & $n=6$ & $n=3$ & & & & Alpha $=0,5$ & Alpha $=0,7$ & Alpha $=0,3$ & \\
\hline 1999 & 734 & 285 & 225 & 198 & 165 & 136 & 164 & 207 & 205 & 206 & 56 \\
\hline 2000 & 770 & 315 & 252 & 226 & 183 & 158 & 180 & 227 & 226 & 227 & 86 \\
\hline 2001 & 772 & 310 & 249 & 224 & 167 & 149 & 162 & 214 & 212 & 213 & 80 \\
\hline 2002 & 847 & 376 & 321 & 304 & 224 & 188 & 216 & 273 & 272 & 273 & 137 \\
\hline 2003 & 865 & 386 & 336 & 320 & 224 & 266 & 214 & 276 & 274 & 276 & 150 \\
\hline
\end{tabular}


Con este método de prueba se mide el Con la suma de todos los errores al cuadrado menor error cuadrático partiendo de la dife- RSE se puede determinar cuál estuvo más rencia entre el dato pronosticado menos la cerca al dato real. Los valores de las diferencias demanda y ese resultado elevado al cuadrado. al cuadrado se muestran a continuación:

\begin{tabular}{|c|c|c|c|c|c|c|c|c|c|c|c|}
\hline \multirow[b]{2}{*}{ Año } & \multirow{2}{*}{$\begin{array}{c}\text { Demanda } \\
\text { real }\end{array}$} & \multicolumn{3}{|c|}{ Media Móvil } & \multirow{2}{*}{$\begin{array}{l}\text { Método } \\
\text { de Brown }\end{array}$} & \multirow{2}{*}{$\begin{array}{l}\text { Método } \\
\text { de } \\
\text { Winters }\end{array}$} & \multirow{2}{*}{$\begin{array}{l}\text { Método } \\
\text { de Holt }\end{array}$} & \multicolumn{3}{|c|}{ Suavización exponencial simple } & \multirow{2}{*}{$\begin{array}{l}\text { Método } \\
\text { Torres }\end{array}$} \\
\hline & & $n=12$ & $n=6$ & $n=3$ & & & & $\begin{array}{c}\text { Alpha }= \\
0,5\end{array}$ & $\begin{array}{c}\text { Alpha }= \\
0,7\end{array}$ & $\begin{array}{c}\text { Alpha }= \\
0,3\end{array}$ & \\
\hline 1999 & 734 & 81.420 & 50.475 & 39.244 & 27.225 & 18.496 & 26.896 & 42.849 & 42.025 & 42.436 & 3.136 \\
\hline 2000 & 770 & 98.912 & 63.476 & 50.956 & 33.489 & 24.964 & 32.400 & 51.529 & 51.076 & 51.529 & 7.396 \\
\hline 2001 & 772 & 95.803 & 61.952 & 50.271 & 27.889 & 22.201 & 26.244 & 45.796 & 44.944 & 45.369 & 6.400 \\
\hline 2002 & 847 & 141.750 & 102.807 & 92.628 & 50.176 & 35.344 & 46.656 & 74.529 & 73.984 & 74.529 & 18.769 \\
\hline 2003 & 865 & 148.769 & 112.924 & 102.462 & 50.176 & 70.756 & 45.796 & 76.176 & 75.076 & 76.176 & 22.500 \\
\hline \multicolumn{2}{|c|}{ Total } & 566.654 & 391.634 & 335.560 & 188.955 & 171.761 & 177.992 & 290.879 & 287.105 & 290.039 & 58.201 \\
\hline
\end{tabular}

Los resultados de la clasificación son los siguientes:

\begin{tabular}{||l|l|r|r||}
\hline Ranking & \multicolumn{1}{|c|}{ Método } & $\begin{array}{c}\text { Suma Errores } \\
\text { cuadrado }\end{array}$ & $\begin{array}{r}\text { Desviación } \\
\text { Porcentual }\end{array}$ \\
\hline Primero & Torres & $\mathbf{5 8 . 2 0 1}$ & $\mathbf{0 , 0 0 \%}$ \\
\hline Segundo & Winters & 171.761 & $195,12 \%$ \\
\hline Tercero & Holt & 177.992 & $205,82 \%$ \\
\hline Cuarto & Brown & 188.955 & $224,66 \%$ \\
\hline Quinto & Suavización Alpha 0,5 & 287.105 & $393,30 \%$ \\
\hline Sexto & Suavización Alpha 0,7 & 290.039 & $398,34 \%$ \\
\hline Séptimo & Suavización Alpha 0,3 & 290.879 & $399,78 \%$ \\
\hline Octavo & Promedio Móvil N = 3 & 335.560 & $476,55 \%$ \\
\hline Noveno & Promedio Móvil N = 6 & 391.634 & $572,90 \%$ \\
\hline Décimo & Promedio Móvil N =12 & 566.654 & $873,62 \%$ \\
\hline
\end{tabular}

\section{CONCLUSIONES}

A partir de cuatro pruebas con colecciones de datos históricos se logró, generar un ranking en cada uno de los casos para comparar los resultados del método de Torres frente otros métodos de suavización.

En dos de las cuatro ocasiones es decir, en el 50 por ciento de los casos el método de
Torres estuvo en el primer lugar superando los nueve métodos restantes. Estuvo por encima de los métodos de Holt, Winters y Brown que forman parte de paquetes computacionales. Este fue un resultado grato para este documento, y para el documento anterior, ya que muestra la validez de las pruebas y la pertinencia de los dos análisis. 
En una de las cuatro ocasiones es decir, en un 25 por ciento de los casos logró el segundo puesto entre los diez métodos estando sólo por debajo del método de Winters, pero nuevamente sobre métodos más convencionales.

Finalmente, en una de las cuatro pruebas es decir, en un 25 por ciento estuvo en quinto lugar viéndose superado por el método de Winters y por los de suavización exponencial simple planteados en las pruebas. En esta prueba estuvo seguido muy de cerca por los métodos de Holt y Brown.

Los resultados de estas pruebas permiten inferir que el método debe ser analizado detenidamente ya que si se usa correctamente es decir, determinando de una manera fiable el \% $\mathrm{P}$ requerido como variación general de la serie se pueden obtener resultados satisfactorios.

Una de las recomendaciones antes de usar el método de Torres es determinar el mejor ajuste usando los diferentes métodos propuestos en el documento: revisión del método de pronóstico para elevar el nivel de precisión del mismo.

Otra recomendación es usar diferentes métodos para verificar el ajuste que tienen con cada una de las series de datos históricos. Esto se logra fácilmente a través de los programas de computador disponibles en el mercado.

\section{BIBLIOGRAFÍA}

- Alarcón, M. (2005). Revisión de método de pronóstico. Bogotá: Politécnico Grancolombiano.

- Fogarty, D. (1999). Administración de la producción e inventarios. México: Editorial CECSA.

- Hiller, F. (2001). Investigación de operaciones. México: Editorial Mc Graw Hill.

- Makridakis, S. (1997). Métodos de pronóstico. México: Editorial Limusa.

- Makridakis, S. (1978). Forecasting Methods and Aplications. México: Editorial Limusa.

- Paredes, J. (1994). Planificación de la producción. México: Editorial Mc Graw Hill.

- El libro completo se puede bajar de: http://bibliotecavirtual.clacso.org.ar/ar/libros/ ecuador/idiuc/teoria.doc. Visitado en septiembre de 2005.

- Pérez López, C. (2005). Métodos estadísticos avanzados con SPSS. Madrid: Editorial Thompson.

- Pindyck, R. (2001). Econometría y modelos de pronóstico. México: Editorial Mc Graw Hill.

- Prawda, J. (1999). Métodos y modelos de investigación de operaciones. México: Editorial Limusa.

- Torres Acosta, J. H. Elementos de producción. Bogotá: Editorial Universidad Católica de Colombia. Sin año de publicación.

- Turoff, M. \& Linstone, H. (2002). The Delphi Method, Techniques and Applications. Boston, Massachusetts. Editorial: Addison Wesley Longman Publishing Co. 
- El libro completo en versión digital se puede bajar de: http://www.is.njit.edu/pubs/ delphibook/index.html. Visitado en octubre 11 de 2005.

- Vélez Pareja, I. (2004). Decisiones empresariales bajo incertidumbre. Bogotá: Editorial Norma.

\section{NOTAS}

1. No se incluyen en la prueba métodos de pronóstico diferentes a los de suavización exponencial, como los ARIMA y los de descomposición, por tener una manera diferente de suavización exponencial. Pero si se quiere probar la precisión del método de Torres en comparación con estos métodos, la prueba presentada serviría para dicho fin.

2. Estas recomendaciones han sido obtenidas de la descripción de los diferentes métodos de pronóstico. Para ampliar estos conceptos ver: Makridakis (1978).

3. Este software tiene un módulo específico para los pronósticos a través de series de tiempo y un apartado para los métodos de suavizado exponencial.

4. En el documento anterior sobre la revisión del método de Torres, se puede ver la manera propuesta de calcular el componente subjetivo de acuerdo con la tendencia que tenga el mayor R2. Si desea ampliar este concepto puede dirigirse a: http://www.poligran.edu.co/documentos

5. American Production and Inventory Control Society. Puede accederse desde: www.apics.org

6. Si se quiere revisar completamente el funcionamiento del método de Torres puede verse: Torres (2000). Si se quiere ver la descripción completa del análisis del método puede verse: Alarcón (2005) desde www. poligran.edu.co/documentos

7. \%P, denota el crecimiento o disminución de la tendencia general de los datos para el siguiente período. Torres menciona este dato como el método Delphi.

8. Se debe aclarar que para reemplazar el dato subjetivo $\% \mathrm{P}$, lo que se hizo fue seguir la metodología del paper Revisión del método de pronóstico, que consiste en usar la línea de tendencia que tenga el mayor R2 (error al cuadrado)17, y extrapolar la tendencia de la serie de datos históricos.

9. RSE hace referencia al valor de la suma de los errores elevados al cuadrado. Para este caso llámese error, al valor de diferencia entre el dato pronosticado y el dato real. Si se desea ampliar el concepto de RSE diríjase a Makridakis, S. (1997). Métodos de pronóstico. México, Editorial Limusa, pp. 75-81.

10. Consumo promedio de kilovatios de energía eléctrica por año. Fuente: Departamento Nacional de Planeación (DNP). Puede accederse a esta serie de datos desde: http://www.dnp.gov.co, en el apartado de estadísticas.

11. Consumo promedio de barriles de gasolina por mes. Fuente: Departamento Nacional de Planeación (DNP). Puede accederse a esta serie de datos desde: http://www.dnp.gov.co, en el apartado de estadísticas.

12. Consumo anual de papel en Colombia desde el año 1974 hasta el año 2003. Fuente: Cámara de Pulpa, Papel y Cartón de la ANDI. Tomado de: Departamento Nacional de Planeación (DNP). Puede accederse a esta serie de datos desde: http://www.dnp.gov.co, en el apartado de estadísticas. 